\title{
Feedback Control in the Presence of Noisy \\ Channels: "Bode-Like" Fundamental \\ Limitations of Performance
}

Nuno C Martins and Munther A Dahleh

\begin{abstract}
This paper addresses a fundamental limitation of performance for feedback systems, in the presence of a communication channel. The feedback loop comprises a discrete-time, linear and time-invariant plant, a channel, an encoder and a decoder which may also embody a controller. Measurements of the plant's output must be encoded for transmission over the channel. Information, at the other end of the channel, is decoded and used to generate a control signal, which is additively disturbed by a Gaussian and stationary stochastic process. We derive an inequality of the form $L_{-} \geq \sum \max \left\{0, \log \left(\left|\lambda_{i}(A)\right|\right)\right\}-$ $C_{\text {channel }}$, where $L_{-}$is a measure of disturbance rejection, $A$ is the open loop dynamic matrix and $C_{\text {channel }}$ is the Shannon capacity of the channel. Our measure $L_{-}$is non-negative and smaller $L_{-}$ indicates better rejection (attenuation), while $L_{-}=0$ signifies no rejection. Previous results show that $C_{\text {channel }}>\sum \max \left\{0, \log \left(\left|\lambda_{i}(A)\right|\right)\right\}$ is a necessary condition for stability and now we show that the extra rate $C_{\text {channel }}-\sum \max \left\{0, \log \left(\left|\lambda_{i}(A)\right|\right)\right\}$ determines a fundamental limitation for disturbance rejection. Additionally, we prove that, under a stationarity assumption, $L_{-}$admits a log-sensitivity integral representation. We contrast our condition with Bode's integral formula and the water-bed effect. The new inequality shows explicitly how the capacity of the channel limits closed loop performance.
\end{abstract}

\section{INTRODUCTION}

Motivated by applications, such as remote feedback, control in the presence of information constraints has received considerable attention. Certainly, the exploration of such problems is

Nuno C Martins and Munther A Dahleh are with the Dept. of Electrical Engineering and Computer Science, Massachusetts Institute of Technology. Address for correspondence: Laboratory for Information and Decision Systems, Room 35-415, Massachusetts Institute of Technology, 77 Mass. Ave, 02139 Cambridge, MA (E-mail:nmartins@mit.edu ) 


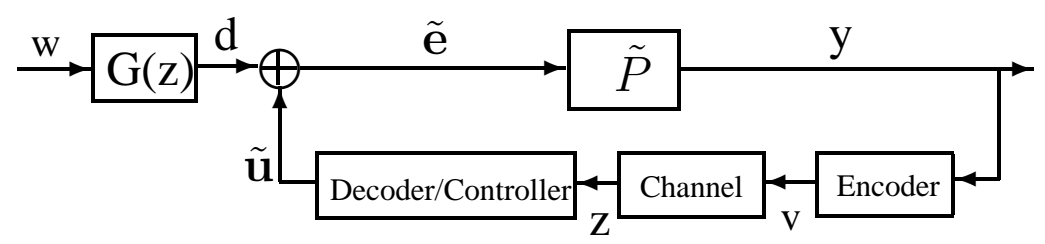

Fig. 1. Structure of the Feedback Interconnection

exciting as they foster the interaction between the disciplines of Information Theory and Control. So far, research in this field has, primarily, directed its attention to stabilization [23]. The basic framework is depicted in Fig 1 and comprises a plant, a channel, an encoder and a decoder, which implicitly embeds a controller. Measurements of the plant's output must be encoded and sent through the channel. The information, received at the other end of the channel, is decoded and used to generate a control signal. It has been shown that stabilization, of a linear and time-invariant plant, requires that the channel's Shannon capacity $C_{\text {channel }}$ is larger than $\sum \max \left\{0, \log \left(\left|\lambda_{i}(A)\right|\right)\right\}$, where $A$ is the dynamic matrix of the state-space representation of the plant [19], [20]. For certain channels, the condition $C_{\text {channel }}>\sum \max \left\{0, \log \left(\left|\lambda_{i}(A)\right|\right)\right\}$ is sufficient for stabilization in the almost sure sense [20], but it may not suffice for moment stability[18]. In general, moment stability necessitates a more informative notion of capacity, designated as Anytime Capacity [18]. Stabilization of nonlinear systems has also been studied by [16] and [11]. The work by [6] has used the integral of the log-sensitivity, as seen by the noise in an additive channel, to establish that encoding/decoding schemes can be constructed using standard optimal control theory. Another recent area of investigation is the analysis in the presence of disturbances and uncertainty. In [13], stability in the presence of disturbances and operator theoretic uncertainty is investigated, for a particular class of channels. The work by [15], has shown that the extra rate $C_{\text {channel }}-\sum \max \left\{0, \log \left(\left|\lambda_{i}(A)\right|\right)\right\}$ is critical for performance, as measured by the expected power of the state of the plant. There, it is proven that the expected power of the state of the plant gets arbitrarily large as $C_{\text {channel }}$ decreases towards the critical value for stabilizability, i.e., $\sum \max \left\{0, \log \left(\left|\lambda_{i}(A)\right|\right)\right\}$.

Understanding the fundamental limitations of performance in a feedback system is critical for effective control design. One of the most well known trade-offs is the water-bet effect for linear feedback systems, which results from Bode's integral formula[3]. In such classical theory, the 
transfer function, between the disturbance $\mathbf{d}$ and $\tilde{\mathbf{e}}=\tilde{\mathbf{u}}+\mathbf{d}$ (see Fig 1), is denoted as sensitivity and is represented by $S(z)$. Bode's result, for a strictly proper loop gain, is expressed as:

$$
\frac{1}{2 \pi} \int_{-\pi}^{\pi}\left[\log \left|S\left(e^{j \omega}\right)\right|\right]_{-} d \omega+\frac{1}{2 \pi} \int_{-\pi}^{\pi}\left[\log \left|S\left(e^{j \omega}\right)\right|\right]_{+} d \omega=\sum \max \left\{0, \log \left(\left|\lambda_{i}(A)\right|\right)\right\}
$$

where $\left[\log \left|S\left(e^{j \omega}\right)\right|\right]_{-}=\min \left\{0, \log \left|S\left(e^{j \omega}\right)\right|\right\}$ and $\left[\log \left|S\left(e^{j \omega}\right)\right|\right]_{+}=\max \left\{0, \log \left|S\left(e^{j \omega}\right)\right|\right\}$. It implies that sensitivity can't be small at all frequencies, i.e., reduction of $\int_{-\pi}^{\pi}\left[\log \left|S\left(e^{j \omega}\right)\right|\right]_{-} d \omega$ is achieved at the expense of increase in $\int_{-\pi}^{\pi}\left[\log \left|S\left(e^{j \omega}\right)\right|\right]_{+} d \omega$.

Recent publications [8], [25] have provided new versions of (1). The work by [25] has introduced a Bode-like integral inequality for non-linear systems, which is derived based on information theoretic principles.

In this paper $^{1}$, we derive a fundamental limitation that arises when the directed information rate $^{2}$ [14], [20], denoted by $\bar{I}_{\infty}(\mathbf{v} \rightarrow \mathbf{z})$, at the channel, is upper-bounded by a constant, i.e., $\bar{I}_{\infty}(\mathbf{v} \rightarrow \mathbf{z}) \leq C_{\text {channel }}$. Our results show that the following must hold:

$$
\frac{1}{2} L_{-}+\bar{I}_{\infty}(\mathbf{v} \rightarrow \mathbf{z}) \geq \sum \max \left\{0, \log \left(\left|\lambda_{i}(A)\right|\right)\right\}
$$

where $L_{-}$is a measure of disturbance rejection. Such measure satisfies $L_{-} \leq 0$, where $L_{-}=0$ means no-rejection and small $L_{-}$attests disturbance attenuation. We show that, under stationarity assumptions, $L_{-}$becomes an integral and our condition can be expressed as:

$$
\frac{1}{2 \pi} \int_{-\pi}^{\pi}\left[\log \left|S\left(e^{j \omega}\right)\right|\right]_{-} d \omega+\bar{I}_{\infty}(\mathbf{v} \rightarrow \mathbf{z}) \geq \sum \max \left\{0, \log \left(\left|\lambda_{i}(A)\right|\right)\right\}
$$

By means of an argument similar to the water-bed effect, the inequality (2) asserts that attenuation, when measured by $\int_{-\pi}^{\pi}\left[\log \left|S\left(e^{j \omega}\right)\right|\right]_{-} d \omega$, has to be repaid by a higher information rate in the channel. Since $\bar{I}_{\infty}(\mathbf{v} \rightarrow \mathbf{z}) \leq C_{\text {channel }}$, we infer that the trade-off (2) creates a fundamental limitation.

Using information theoretic arguments and assuming stationarity, we also derive the Bode integral formula. Our derivations require a linear and time-invariant plant, but the encoder, the channel and the decoder/controller can be any causal operators. The paper is organized in 4 sections. Besides the introduction, section II lays down the problem formulation as well as a preview and a discussion of the results; the limitations resulting from causality are derived in

\footnotetext{
${ }^{1}$ This paper is identical to Chapter 3 of the Ph.D. Thesis [12]. The results date back to the defense date: May 2004.

${ }^{2}$ This quantity is represented as $\bar{I}_{\infty}(\mathbf{v} \rightarrow \mathbf{z})$ and will be precisely defi ned in section II.
} 
section III and section IV develops a fundamental limitation that results from finite capacity feedback.

\section{A. The following notation is adopted:}

- Whenever it is clear from the context, we refer to a sequence $\{a(k)\}_{-\infty}^{\infty}$ of elements in $\mathbb{R}^{n}$ as $a$. A finite segment of a sequence $a$ is indicated as $a_{k_{\min }}^{k_{\max }}=\{a(k)\}_{k_{\min }}^{k_{\max }}$. If $k_{\max }<k_{\min }$ then $a_{k_{\min }}^{k_{\max }}=\emptyset$.

- If $\mathcal{O} \subset \mathbb{R}^{q}$ is a Borel set then we denote its volume by $\operatorname{Vol}(\mathcal{O})$

- If $M$ is a matrix then the element in the $i$-th row and $j$-th column is indicated as $[M]_{i, j}$. Similarly, if $a \in \mathbb{R}^{n}$ then $[a]_{i}$ denotes the $i$-th component of the vector.

- Random variables are represented using boldface letters, such as a.

- If $\mathbf{a}(k)$ is a stochastic process, then we use $a(k)$ to indicate a specific realization. Similar to the convention used for sequences, we may denote $\mathbf{a}(k)$ just as a and $a(k)$ as $a$. A finite segment of a stochastic process is indicated as $\mathbf{a}_{k_{\min }}^{k_{\max }}$.

- The probability density of a random variable $\mathbf{a}$, if it exists, is denoted as $p_{a}$. The conditional probability, given $\mathbf{b}$, is indicated as $p_{a \mid b}$.

- The expectation operator over a is written as $\mathcal{E}[\mathbf{a}]$

- We write $\log _{2}($.$) simply as \log ($.

- We adopt the convention $0 \log 0=0$

- The auto-covariance function of a given stochastic process a is given by:

$$
R_{a}(k, l)=\mathcal{E}\left[(\mathbf{a}(k)-\mathcal{E}[\mathbf{a}(k)])(\mathbf{a}(l)-\mathcal{E}[\mathbf{a}(l)])^{T}\right]
$$

If $\mathbf{a}$ is stationary then it's power spectral density is written as

$$
\hat{F}_{a}(\omega)=\sum_{k=-\infty}^{\infty} R_{a}(k, 0) e^{-i \omega k}
$$

- If $\mathbf{a}$ is a stochastic process taking values in $\mathbb{R}$ then we use the following covariance matrix:

$$
\left[\Sigma\left(\mathbf{a}_{k_{\min }}^{k_{\max }}\right)\right]_{\left(i-k_{\min }+1\right),\left(j-k_{\min }+1\right)}=\mathcal{E}[(\mathbf{a}(i)-\mathcal{E}[\mathbf{a}(i)])(\mathbf{a}(j)-\mathcal{E}[\mathbf{a}(j)])]
$$

where $i, j \in\left\{k_{\min }, \ldots, k_{\max }\right\}$.

- The Singular Value Decomposition of a matrix $M=M^{H} \geq 0$ is indicated as $M=$ $V_{M}^{T} \Lambda_{M} V_{M}$, where the usual ordering of singular values is assumed $\left[\Lambda_{M}\right]_{i+1, i+1} \leq\left[\Lambda_{M}\right]_{i, i}$. 
The singular values of $M$ are represented in a more streamlined form as $\lambda_{i}(M)=\left[\Lambda_{M}\right]_{i, i}$. If $A$ is a square matrix, we also represent its eigenvalues as $\lambda_{i}(A)$.

- If $a \in \mathbb{R}$ then we define the negative and positive parts of $a$ as $[a]_{-}=\min \{a, 0\}$ and $[a]_{+}=\max \{a, 0\}$, respectively.

- The following is a shorthand notation for the log-density of the eigenvalues with magnitude smaller than 1 , of a covariance matrix:

$$
L_{-}\left(\mathbf{a}_{k_{\min }}^{k_{\max }}\right)=\frac{1}{k_{\max }-k_{\min }+1} \sum_{i=1}^{k_{\max }-k_{\min }+1}\left[\log \left(\lambda_{i}\left(\Sigma\left(\mathbf{a}_{k_{\min }}^{k_{\max }}\right)\right)\right]_{-}\right.
$$

Similarly, we also define the positive counterpart of $L_{-}$as:

$$
L_{+}\left(\mathbf{a}_{k_{\min }}^{k_{\max }}\right)=\frac{1}{k_{\max }-k_{\min }+1} \sum_{i=1}^{k_{\max }-k_{\min }+1}\left[\log \left(\lambda_{i}\left(\Sigma\left(\mathbf{a}_{k_{\min }}^{k_{\max }}\right)\right)\right]_{+}\right.
$$

\section{B. Basic Facts and Definitions of Information Theory}

In this section, we summarize the main definitions and facts from Information Theory which are used throughout the paper. We adopt [17], as a primary reference, because it addresses general probabilistic spaces in a unified framework. Let $\left(\Omega, \mathcal{S}_{\omega}, \mathcal{P}_{\omega}\right)$ be a probability space along with random variables $\mathbf{a}, \mathbf{b}$ and $\mathbf{c}$, taking values in the measurable spaces $\left(\mathcal{A}, \mathcal{S}_{a}\right),\left(\mathcal{B}, \mathcal{S}_{b}\right)$ and $\left(\mathcal{C}, \mathcal{S}_{c}\right)$. We define mutual information and conditional mutual information, between any two random variables, as:

Definition 1.1: (from [17] pp. 9 ) The mutual information, between $\mathbf{a}$ and $\mathbf{b}, I:(\mathbf{a} ; \mathbf{b}) \rightarrow$ $\mathbf{R}_{+} \bigcup\{\infty\}$ is given by:

$$
I(\mathbf{a} ; \mathbf{b})=\sup \sum_{i j} \mathcal{P}_{\mathbf{a}, \mathbf{b}}\left(E_{i} \times F_{j}\right) \log \frac{\mathcal{P}_{\mathbf{a}, \mathbf{b}}\left(E_{i} \times F_{j}\right)}{\mathcal{P}_{\mathbf{a}}\left(E_{i}\right) \mathcal{P}_{\mathbf{b}}\left(F_{j}\right)}
$$

where the supremum is taken over all partitions $\left\{E_{i}\right\}$ of $\mathcal{A}$ and $\left\{F_{j}\right\}$ of $\mathcal{B}$.

Definition 1.2: (from [17] pp. 37 ) The conditional mutual information between a and b given c, is defined as:

$$
I(\mathbf{a} ; \mathbf{b} \mid \mathbf{c})=\sup \sum_{i j k} \mathcal{P}_{\mathbf{a}, \mathbf{b}, \mathbf{c}}\left(E_{i} \times F_{j} \times N_{k}\right) \log \frac{\mathcal{P}_{\mathbf{a}, \mathbf{b}, \mathbf{c}}\left(E_{i} \times F_{j} \times N_{k}\right)}{\overline{\mathcal{P}}_{\mathbf{a}, \mathbf{b} \mid \mathbf{c}}\left(E_{i} \times F_{j} \times N_{k}\right)}
$$

where the supremum is taken over all partitions $E_{i} \in \mathcal{A}, F_{j} \in \mathcal{B}$ and $N_{k} \in \mathcal{C}$ and $\overline{\mathcal{P}}_{\mathbf{a}, \mathbf{b} \mid \mathbf{c}}$ is given by:

$$
\overline{\mathcal{P}}_{\mathbf{a}, \mathbf{b} \mid \mathbf{c}}(E \times F \times N)=\int_{N} \mathcal{P}_{\mathbf{a} \mid \mathbf{c}}(E \mid \gamma) \mathcal{P}_{\mathbf{b} \mid \mathbf{c}}(F \mid \gamma) P_{\mathbf{c}}(d \gamma)
$$


Notice that, in definition $1.1, \mathcal{A}$ and $\mathcal{B}$ may be different.

Consistent with the usual notation [17], [4], we define entropy as:

Definition 1.3: (Entropy) Let $\mathbf{a}$ and $\mathbf{b}$ be random variables. The entropy of $\mathbf{a}$ given $\mathbf{b}$ is defined as:

$$
H(\mathbf{a} \mid \mathbf{b})=I(\mathbf{a} ; \mathbf{a} \mid \mathbf{b})
$$

Since entropy may be infinite for random variables defined in continuous probability spaces, we also define the following quantities, denoted as differential entropy and conditional differential entropy.

Definition 1.4: If a is a random variable, where $\mathcal{A}=\mathbb{R}^{q}$, along with a Lebesgue measurable and bounded probability density function $p_{a}(\cdot)$ then we define the differential entropy of a as:

$$
h(\mathbf{a})=\int_{p_{a}(\gamma) \leq 1}-p_{a}(\gamma) \log p_{a}(\gamma) d \gamma-\int_{p_{a}(\gamma)>1} p_{a}(\gamma) \log p_{a}(\gamma) d \gamma
$$

Notice that if $p_{a}$ is Lebesgue measurable and bounded then we use $\int_{p_{a}(\gamma)>1} p_{a}(\gamma) \leq 1$ to assert that $\int_{p_{a}(\gamma)>1} p_{a}(\gamma) \log p_{a}(\gamma) d \gamma<\infty$. This implies that $h(\mathbf{a})$ is always well defined, although not necessarily bounded. Consequently, a bounded Lebesgue measurable $p_{a}$ leads to an almostintegrable $p_{a}(\gamma) \log \left(p_{a}(\gamma)\right)$.

If $\mathbf{b}$ is another random variable and $I(\mathbf{a}, \mathbf{b})<\infty$ then the conditional differential entropy of a given $\mathbf{b}$ is defined by:

$$
h(\mathbf{a} \mid \mathbf{b})=h(\mathbf{a})-I(\mathbf{a} ; \mathbf{b})
$$

For technical reasons, we also define the following class of random variables:

Definition 1.5: (Dither Class denoted as $\mathbb{D}$ ) Let $\mathbf{b}$ be a random variable with alphabet $\mathcal{B} \subset \mathbf{R}^{q}$, for some $q \in \mathbb{N}$. We denote $\mathbf{b}$ as type 1 if it has a countable alphabet with $\inf \left\{\max _{i}\left|b_{i}-\tilde{b}_{i}\right|\right.$ : $b, \tilde{b} \in \mathcal{B}, b \neq \tilde{b}\}>0$ and type 2 if it has a probability density $p_{b}$ which is Lebesgue measurable. The random variable $\mathbf{b}$ is of the class Dither, denoted as $\mathbb{D}$, if it is type 1 or type 2 . If $\mathbf{b} \in \mathbb{D}$ then we also define its dithered version $\breve{b}$ as:

$$
\breve{\mathbf{b}}= \begin{cases}\mathbf{b}+\mathbf{s} \Delta & \text { if } \mathbf{b} \text { is type } 1 \\ \mathbf{b} & \text { if } \mathbf{b} \text { is type } 2\end{cases}
$$

\footnotetext{
${ }^{3}$ According to [7], all the properties of Lebesgue integrable functions hold for almost-integrable functions. A Lebesgue measurable function $f$ on $(\mu, X)$ is almost integrable [7] if at least one of the following holds: $\int_{X}[f]_{+} \mu(d x)<\infty$ or $\int_{X}[-f]_{+} \mu(d x)<\infty$. The integral of an almost integrable $f$ is defi ned as $\int_{X} f \mu(d x)=\int_{X}[f]_{+} \mu(d x)-\int_{X}[-f]_{+} \mu(d x)$. This issue is also briefly discussed in pp. 200 of [2].
} 
where $\mathbf{s}$ is a random variable, which is independent of $\mathbf{b}$ and uniformly distributed in $(-1 / 2,1 / 2)^{q}$. The constant $\Delta$ is given by:

$$
\Delta=\inf \left\{\max _{i}\left|b_{i}-\tilde{b}_{i}\right|: b, \tilde{b} \in \mathcal{B}, b \neq \tilde{b}\right\}
$$

Notice that $\Delta$ is such that the following projections always exist:

$$
\begin{gathered}
b=\pi_{\mathcal{B}}(\breve{b}) \\
\pi_{\mathcal{S}}(\breve{b})= \begin{cases}\mathbf{s} & \text { if } \mathbf{b} \text { is type } 1 \\
0 & \text { if } \mathbf{b} \text { is type } 2\end{cases}
\end{gathered}
$$

The following is a list of properties used in the sections III and IV. The proof of such properties may be found in [17] and, in some cases, in [4]. We emphasize that, in this paper, we write $h(\mathbf{a} \mid \cdot)$ only if the assumptions stated in definition 1.4 are satisfied.

- (P1): $I(\mathbf{a} ; \mathbf{b})=I(\mathbf{b} ; \mathbf{a}) \geq 0$ and $I(\mathbf{a} ; \mathbf{b} \mid \mathbf{c})=I(\mathbf{b} ; \mathbf{a} \mid \mathbf{c}) \geq 0$

- (P2) Kolmogorov's formula ${ }^{4}$ (equation 3.6.6 in [17]):

$$
I((\mathbf{a}, \mathbf{b}) ; \mathbf{c} \mid \mathbf{d})=I(\mathbf{b} ; \mathbf{c} \mid \mathbf{d})+I(\mathbf{a} ; \mathbf{c} \mid(\mathbf{b}, \mathbf{d}))
$$

- (P3) Theorem 3.7.1 in [17]: If $f$ and $g$ are measurable functions then $I(f(\mathbf{a}) ; g(\mathbf{b}) \mid \mathbf{c}) \leq$ $I(\mathbf{a} ; \mathbf{b} \mid \mathbf{c})$ and equality holds ${ }^{5}$ if $f$ and $g$ are invertible.

- (P3') It follows from (P3) that if $\mathbf{b} \in \mathbb{D}$ then $I(\mathbf{a} ; \breve{\mathbf{b}} \mid \mathbf{c})=I(\mathbf{a} ;(\mathbf{b}, \mathbf{s}) \mid \mathbf{c})$ which, since $\mathbf{s}$ is independent from the rest, also implies that $I(\mathbf{a} ; \breve{\mathbf{b}} \mid \mathbf{c})=I(\mathbf{a} ; \mathbf{b} \mid \mathbf{c})$. A similar argument, using $(P 2)$, also leads to $I(\mathbf{a} ; \mathbf{b} \mid \mathbf{c})=I(\mathbf{a} ; \mathbf{b} \mid \breve{\mathbf{c}})$, provided that $\mathbf{c} \in \mathbb{D}$.

- (P4) Corollary 2., pp. 43 in [17]: Given a function $f: \mathcal{C} \rightarrow \mathcal{C}^{\prime}$ it follows that $I(\mathbf{a} ; f(\mathbf{c}) \mid \mathbf{c})=$ 0 .

-(P5): From property (P3), we conclude that $I(\mathbf{a} ;(\mathbf{b}, \mathbf{c}) \mid \mathbf{d})=I(\mathbf{a} ;(\mathbf{b}-\mathbf{c}, \mathbf{c}) \mid \mathbf{d})$. Using (P2), such equality also leads to:

$$
I(\mathbf{a} ; \mathbf{b} \mid(\mathbf{c}, \mathbf{d}))=I(\mathbf{a} ; \mathbf{b}-\mathbf{c} \mid(\mathbf{c}, \mathbf{d}))
$$

${ }^{4}$ Notice that equation 3.6.3 in [17] has a typographic mistake. On the left hand side of the equality, the correct is $I(\xi, \zeta)$

${ }^{5}$ The general version of this property states that equality holds if $f$ and $g$ are everywhere dense [17]. Every-time we use an invertible function to claim equality in (P3) the function is everywhere dense. 
- (P6): By means of (P1) and (3), we infer that $h(\mathbf{a}) \geq h(\mathbf{a} \mid \mathbf{b})$, where equality holds if and only if $\mathbf{a}$ and $\mathbf{b}$ are independent. Likewise, we can use properties (P1)-(P2) to state that $I(\mathbf{a} ;(\mathbf{b}, \mathbf{c})) \geq I(\mathbf{a} ; \mathbf{b})$, which can be used with (3) to derive $h(\mathbf{a} \mid \mathbf{b}) \geq h(\mathbf{a} \mid(\mathbf{b}, \mathbf{c}))$.

- (P7) [4]: Let a, with $\mathcal{A}=\mathbb{R}^{q}$, be a random variable with $p_{a}$ bounded and Lebesgue measurable and a covariance matrix denoted as $\Sigma_{a}$. In Proposition 3.1, of appendix III, we show that finite $\Sigma_{a}$ implies finite $h(\mathbf{a})$. Under such hypothesis, $p_{a}(\gamma) \log \left(p_{a}(\gamma)\right)$ is Lebesgue integrable and the following holds[4]:

$$
h(\mathbf{a}) \leq \frac{1}{2} \log \left((2 \pi e)^{n} \operatorname{det}\left(\Sigma_{a}\right)\right)
$$

where equality holds if $\mathbf{a}$ is Gaussian.

In order to simplify our notation, we also define the following quantities:

Definition 1.6: Let $\mathbf{a}$ and $\mathbf{b}$ be stochastic processes. The following are useful limit information rates:

$$
\bar{I}_{\infty}(\mathbf{a} ; \mathbf{b})=\limsup _{k \rightarrow \infty} \frac{I\left(\mathbf{a}_{1}^{k} ; \mathbf{b}_{1}^{k}\right)}{k}, \bar{I}_{\infty}(\mathbf{a} \rightarrow \mathbf{b})=\limsup _{k \rightarrow \infty} \frac{I\left(\mathbf{a}_{1}^{k} \rightarrow \mathbf{b}_{1}^{k}\right)}{k}
$$

where $I\left(\mathbf{a}_{1}^{k} \rightarrow \mathbf{b}_{1}^{k}\right)$ is denoted as directed mutual information [14], [20] and is defined as:

$$
I\left(\mathbf{a}_{1}^{k} \rightarrow \mathbf{b}_{1}^{k}\right)=\sum_{i=1}^{k} I\left(\mathbf{a}_{1}^{i} ; \mathbf{b}(i) \mid \mathbf{b}_{1}^{i-1}\right)
$$

In this paper, we will also refer to Channels which are stochastic operators conforming to the following definition:

Definition 1.7: (Memory-less Channel) Let $\mathcal{V}$ and $\mathcal{Z}$ be given input and output alphabets, along with a white stochastic process, denoted as $\mathbf{c}$, with alphabet $\mathcal{C}$. Consider $f: \mathcal{V} \times \mathcal{C} \rightarrow \mathcal{Z}$ such that the following maps are invertible:

$$
\begin{aligned}
& g_{1}(v(k), c(k))=(v(k), f(v(k), c(k))) \\
& g_{2}(v(k), c(k))=(f(v(k), c(k)), c(k))
\end{aligned}
$$

The pair $(f, \mathbf{c})$ defines a memory-less channel.

The previous definition is sufficiently general to encompass the following examples:

- Additive white Gaussian channel: $\mathcal{V}=\mathcal{Z}=\mathcal{C}=\mathbb{R}$, $\mathbf{c}$ is an i.i.d. white Gaussian sequence with unit variance and $f(c, v)=c+v$.

- Binary symmetric channel, with error probability $p_{e}: \mathcal{V}=\mathcal{Z}=\mathcal{C}=\mathbb{Z}_{2}=\{0,1\}$, c is an i.i.d sequence satisfying $\mathcal{P}(\mathbf{c}(k)=1)=p_{e}$ and $f(c, v)=c+{ }_{\bmod 2} v$ 


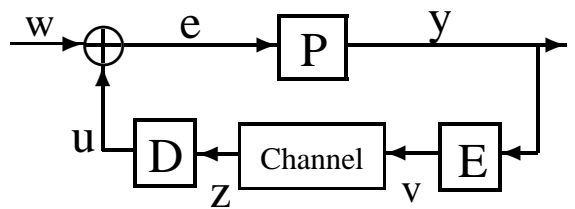

Fig. 2. Simplifi ed Structure of the Feedback Interconnection using $e=G^{-1} \tilde{e}$ and $u=G^{-1} \tilde{u}$, with the correspondence, relative to the blocks of Fig 1 .

\section{Problem Formulation and Discussion of Results}

Consider the feedback interconnection depicted in Figure 1. In such information pattern [21], measurements of the state of the plant have to be encoded and sent over a communication channel. The transmitted information is used, at the decoder/controller, to generate the control signal $\mathbf{u}$. In order to simplify the presentation, we proceed with the equivalent block diagram of Fig 2.

\section{A. Assumptions}

Before stating our assumptions, we need the following definitions:

Definition 2.1: We define the following set of probability densities:

$$
\overline{\mathbb{L}}^{q}=\left\{f: \mathbb{R}^{q} \rightarrow \mathbb{R}_{\geq 0} \mid f \text { is Leb. meas., } \int f(\gamma) d \gamma=1, \int f(\gamma) \gamma^{T} \gamma d \gamma<\infty, \sup _{\gamma} f(\gamma)<\infty\right\}
$$

In addition, we also define:

$$
\mathbb{L}^{q}=\left\{f \in \overline{\mathbb{L}}^{q} \mid \exists \epsilon>0, \text { such that } \Gamma_{f}^{\epsilon} \text { has limited interior }\right\}
$$

where

$$
\Gamma_{f}^{\epsilon}=\left\{\gamma \in \mathbb{R}^{q}: f(\gamma)>\frac{1}{\left(\left(1+\left|\gamma_{1}\right|\right) \cdots\left(1+\left|\gamma_{q}\right|\right)\right)^{1+\epsilon}}\right\}
$$

An important property of $\mathbb{L}^{q}$ is that if $p_{a, b} \in \mathbb{L}^{q_{a}+q_{b}}$ then $p_{a} \in \overline{\mathbb{L}}^{q_{a}}$ and $p_{b} \in \overline{\mathbb{L}}^{q_{b}}$.

In the present formulation, which is schematically depicted in Fig 2, the following assumptions are made. Notice that the diagram in Fig 2 is derived from Fig 1, by means of incorporating $G$ in the plant and $G^{-1}$ in the decoder. The Appendix I comprises a discussion of several important aspects related to the assumptions made.

We adopt the following assumptions: 
- (A1): w, with $w(k) \in \mathbb{R}$, is an i.i.d., zero mean, unit variance and white Gaussian process.

- (A2): the control signal satisfies $\mathbf{u}_{1}^{k} \in \mathbb{D}$ for every $\mathrm{k}$. We denote the alphabet of $\mathbf{u}$ as $\mathcal{U} \subset \mathbf{R}$, so that $u_{1}^{k} \in \mathcal{U}^{k}$. According to definition 1.5, we indicate the dithered version of $\mathbf{u}(k)$ as $\breve{\mathbf{u}}(k)$.

- (A3): $G(z)$ is an all-pole stable filter of the form:

$$
G(z)=\frac{\alpha}{1-\sum_{m=1}^{p} a_{m} z^{-m}}
$$

for some integer $p \geq 1$ and constants $a_{i}$ and $\alpha>0$.

- (A4): given $n, P$ is a single input plant with state $x(k) \in \mathbb{R}^{n}$, which satisfies the following state-space equation:

$$
\begin{gathered}
\mathbf{x}(k+1)=\left[\begin{array}{l}
x_{u}(k+1) \\
x_{s}(k+1)
\end{array}\right]=\left[\begin{array}{cc}
A_{u} & 0 \\
0 & A_{s}
\end{array}\right] \mathbf{x}(k)+\left[\begin{array}{l}
b_{u} \\
b_{s}
\end{array}\right] \mathbf{e}(k) \\
\mathbf{y}(k)=C \mathbf{x}(k),\left|\lambda_{i}\left(A_{u}\right)\right| \geq 1,\left|\lambda_{i}\left(A_{s}\right)\right|<1
\end{gathered}
$$

The state partitions $\mathbf{x}_{u}$ and $\mathbf{x}_{s}$ represent the unstable and stable open-loop dynamics, respectively. In addition, if $A \neq A_{s}$ then $\mathbf{x}_{u}(k)$ is a random variable, with a given probability density $p_{x_{u}(k)}(\cdot)$.

- (A5): the capacity of a channel [4], specified by $(f, \mathbf{c})$, is denoted as $C_{\text {channel }}$ and is defined as:

$$
C_{\text {channel }}=\sup _{P_{v}} I(f(\mathbf{v}(k), \mathbf{c}) ; \mathbf{v}(k))<\infty
$$

where the supremum is taken over all probability measures $P_{v}$, defined in $\left(\mathcal{V}, \mathcal{S}_{v}\right)$.

- (A6): the encoder and the decoder are causal operators defined in the appropriate spaces, i.e., $E: \mathcal{Y}^{\infty} \rightarrow \mathcal{V}^{\infty}, D: \mathcal{Z}^{\infty} \rightarrow \mathcal{U}^{\infty}$ where $\mathbf{v}(k)=f_{k}^{e}\left(\mathbf{y}_{-\infty}^{k}\right)$ and $\mathbf{u}(k)=f_{k}^{d}\left(\mathbf{z}_{-\infty}^{k}\right)$ for some functions $f_{k}^{e}$ and $f_{k}^{d}$.

- (A7): additionally, the decoder satisfies the following finite memory condition:

$$
\forall k>\alpha, \mathbf{u}_{1+\alpha}^{k}=\tilde{f}_{k}^{d}\left(\mathbf{u}_{1}^{\alpha}, \mathbf{z}_{1}^{k}\right)
$$

for some $\alpha \in \mathbb{N}_{+}$and a sequence of functions $\tilde{f}_{k}^{d}: \mathcal{U}^{\alpha} \times \mathcal{Z}^{k} \rightarrow \mathcal{U}^{k-\alpha-1}$.

- (A8):(Fading memory condition) For technical reasons, we assume that the following condition holds:

$$
\limsup _{k \rightarrow \infty} \frac{1}{k} I\left(\mathbf{u}_{1}^{\alpha} ; \mathbf{x}(1), \mathbf{w}_{1}^{k} \mid \mathbf{z}_{1}^{k}\right)=0
$$


where $\alpha$ is the smallest constant for which (A7) holds. If $\alpha=0$ then we adopt the convention that (A8) is satisfied. Several aspects of this assumption are clarified in appendix I. In particular, this condition is automatically satisfied if $\mathcal{U}$ is countable and $H\left(\mathbf{u}_{1}^{\alpha}\right)<\infty$ holds.

- (A9): We assume that, for each $k,\left(\mathbf{w}_{1}^{k}, \breve{\mathbf{u}}_{1}^{k}, \mathbf{x}(1)\right)$ admits a probability distribution satisfying $p_{w_{1}^{k}, \breve{u}_{1}^{k}, x(1)} \in \mathbb{L}^{2 k+1}$. In appendix I, we explore a few special cases related to this assumption.

\section{B. Problem Statement and Summary of Results}

We investigate the fundamental limitations of the asymptotic eigenvalue distribution of $\Sigma\left(\mathbf{e}_{g(k)}^{k}\right)$, where $g: \mathbb{N}_{+} \rightarrow \mathbb{N}_{+}$is any arbitrary function satisfying:

$$
\lim _{k \rightarrow \infty} \frac{g(k)}{k}=0
$$

Under the assumption of asymptotic stationarity, the use of an increasing $g(k)$, instead of $g(k)=1$, will enable the derivation of integral formulae. Under stationarity assumptions, integral formulae can be derived for $g(k)=1$. In order to simplify the exposé, we state our results in terms of $L_{-}\left(\mathbf{e}_{g(k)}^{k}\right)$ and $L_{+}\left(\mathbf{e}_{g(k)}^{k}\right)$.

In section III, we reach a fundamental limitation which is a consequence of causality alone. The result is presented in theorem 3.3, which states that if the feedback system in Fig 2 is stable then the following must hold ${ }^{6}$ :

$$
\frac{1}{2} \liminf _{k \rightarrow \infty}\left(L_{-}\left(\mathbf{e}_{1}^{k}\right)+L_{+}\left(\mathbf{e}_{1}^{k}\right)\right) \geq \sum_{i} \max \left\{0, \log \left(\left|\lambda_{i}(A)\right|\right)\right\}
$$

The inequality in (11) demonstrates that, in the limit, not all of the eigenvalues, of $\Sigma\left(\mathbf{e}_{1}^{k}\right)$, can be made small and that the reduction of some necessarily imply the increase of others. That is comparable to the water-bed effect, associated to the classic Bode integral limitation. Such comparison is not coincidental and is explored in section III-A.

In the fundamental limitation expressed in (11), the characteristics of the channel do not play a role. It remains the question of whether the "shaping" of the eigenvalues of $\Sigma\left(\mathbf{e}_{g(k)}^{k}\right)$ depends on the information flow in the feedback loop. The answer is given in theorem 4.3, which states that:

$$
\frac{1}{2} \liminf _{k \rightarrow \infty} L_{-}\left(\mathbf{e}_{g(k)}^{k}\right)+\bar{I}_{\infty}(\mathbf{v} \rightarrow \mathbf{z}) \geq \sum_{i} \max \left\{0, \log \left(\left|\lambda_{i}(A)\right|\right)\right\}
$$

\footnotetext{
${ }^{6}$ Since this limitation is not the main objective of this paper, we restrict the result for $g(k)=1$. The extension of this inequality, to arbitrary $g(k)$, requires extra assumptions.
} 
where $g: \mathbb{N}_{+} \rightarrow \mathbb{N}_{+}$is any arbitrary function satisfying:

$$
\lim _{k \rightarrow \infty} \frac{g(k)}{k}=0
$$

As a consequence of (12), we find that reduction of the eigenvalues of $\Sigma\left(\mathbf{e}_{g(k)}^{k}\right)$, for values bellow unity, must come at the expense of information flow in the channel, as quantified by $\bar{I}_{\infty}(\mathbf{v} \rightarrow \mathbf{z})$.

Under stationary assumptions, corollaries 3.4 and 4.4 show that the inequalities (11) and (12) can be expressed as:

$$
\begin{gathered}
\frac{1}{2 \pi} \int_{-\pi}^{\pi}[\log (S(\omega))]_{-} d \omega+\frac{1}{2 \pi} \int_{-\pi}^{\pi}[\log (S(\omega))]_{+} d \omega \geq \sum_{i} \max \left\{0, \log \left(\left|\lambda_{i}(A)\right|\right)\right\} \\
\frac{1}{2 \pi} \int_{-\pi}^{\pi}[\log (S(\omega))]_{-} d \omega+\bar{I}_{\infty}(\mathbf{v} \rightarrow \mathbf{z}) \geq \sum_{i} \max \left\{0, \log \left(\left|\lambda_{i}(A)\right|\right)\right\}
\end{gathered}
$$

where $S(\omega)=\sqrt{\hat{F}_{\mathbf{e}}(\omega)}=\sqrt{\frac{\hat{F}_{\hat{e}}(\omega)}{\left|G\left(e^{j \omega}\right)\right|^{2}}}$.

The inequalities (14) and (15) must be satisfied by any stable and causal loop of the form depicted in Fig 1 or Fig 2. The first inequality is the Bode integral formula ${ }^{7}$, which is the basis of the disturbance attenuation/amplification water-bed effect, while the second entails a new attenuation/capacity trade-off.

\section{Fundamental Limitations CReated by CAuSAlity}

In this section, we derive a fundamental limitation that arises from causality. The results are valid under the assumptions listed in section II-A, with the exception of (A7)-(A8) which are not needed. We also introduce some of the preliminary results which will be used in section IV. Our technique follows the one by [25], with the exception of the way we tackle initial conditions and unstable modes of the plant. More specifically, theorem 3.3 states a fundamental limitation that explicitly incorporates the eigenvalues of $A$. At the end of the section, we specialize the result, under stationarity assumptions, and derive the Bode-Integral formula in Corollary 3.4.

The following lemma shows that the difference, between the entropy rate of e and the entropy rate of $\mathbf{w}$, is lower-bounded by the mutual information between the plant's state and $\mathbf{e}$.

\footnotetext{
${ }^{7}$ Bode's integral inequality was extended by Prof. John Doyle (CALTECH), in an yet unpublished work, for general feedback interconnections in a deterministic setting.
} 
Lemma 3.1: (Entropy-rate amplification) If $\mathbf{x}(k)$ is the solution of the state-space equation (8) then the following holds:

$$
\liminf _{k \rightarrow \infty} \frac{h\left(\mathbf{e}_{1}^{k}\right)}{k} \geq \liminf _{k \rightarrow \infty} \frac{I\left(\mathbf{e}_{1}^{k} ; \mathbf{x}(1)\right)}{k}+h(\mathbf{w}(1))
$$

Proof: We start by noticing that, since the plant is strictly proper and causal, w $(k)$, with $k \geq 1$, is independent of $\left(\mathbf{x}(1), \mathbf{u}_{1}^{k}, \mathbf{w}_{1}^{k-1}\right)$, which implies:

$$
h(\mathbf{w}(k))=h\left(\mathbf{w}(k) \mid \mathbf{x}(1), \mathbf{u}_{1}^{k}, \mathbf{w}_{1}^{k-1}\right)=h\left(\mathbf{e}(k) \mid \mathbf{x}(1), \mathbf{u}_{1}^{k}, \mathbf{e}_{1}^{k-1}\right) \leq h\left(\mathbf{e}(k) \mid \mathbf{x}(1), \mathbf{e}_{1}^{k-1}\right), k \geq 1
$$

where we used properties (P6) and lemma 3.3 of appendix III. Since $h(\mathbf{w}(k))$ does not depend on $k$, we use (17) and the chain rule of differential entropy to derive:

$$
\sum_{i=1}^{k} h\left(\mathbf{e}(i) \mid \mathbf{x}(1), \mathbf{e}_{1}^{i-1}\right)=h\left(\mathbf{e}_{1}^{k} \mid \mathbf{x}(1)\right) \geq h(\mathbf{w}(1))
$$

Notice that the chain rule of differential entropy in (18) is valid because $p_{w_{1}^{k}, u_{1}^{k}, x(1)} \in \mathbb{L}^{2 k+1}$ implies, using a change of variables and an integration argument, that the marginal densities ${ }^{8}$ $p_{e_{1}^{i}, x(1)} \in \overline{\mathbb{L}}^{i+1}$. Consequently, the proposition 3.1 and the lemma 3.2, of appendix III, guarantee that all the quantities in (18) are well defined. The proof is concluded once we notice, from (3), that $h\left(\mathbf{e}_{1}^{k} \mid \mathbf{x}(1)\right)=h\left(\mathbf{e}_{1}^{k}\right)-I\left(\mathbf{e}_{1}^{k} ; \mathbf{x}(1)\right)$.

The following lemma, corroborates the results by [19], [20], [18], [24], [15], and unveils that stability implies that e must carry a bit-rate of information about the state of the plant, of at least $\sum_{i} \max \left\{0, \log \left(\left|\lambda_{i}(A)\right|\right)\right\}$.

Lemma 3.2: Let $\mathrm{x}(k)$ be the solution of the state-space equation (8). If the plant is stabilized, i.e., $\sup _{k} \mathcal{E}\left[\mathbf{x}^{T}(k) \mathbf{x}(k)\right]<\infty$ holds then the following is satisfied:

$$
\liminf _{k \rightarrow \infty} \frac{I\left(\mathbf{e}_{1}^{k} ; \mathbf{x}(1)\right)}{k} \geq \sum_{i} \max \left\{0, \log \left(\left|\lambda_{i}(A)\right|\right)\right\}
$$

Proof: If $A=A_{s}$ then we just use $I\left(\mathbf{e}_{1}^{k} ; \mathbf{x}(1)\right) \geq 0$. If $A \neq A_{s}$ then we consider the following homogeneous system:

$$
\mathbf{x}_{e}(k+1)=A_{u} \mathbf{x}_{e}(k)+b_{u} \mathbf{e}(k), x_{e}(1)=0
$$

\footnotetext{
${ }^{8}$ Notice that Fubinni's Theorem [1] guarantees that the marginal densities are Lebesgue measurable. The co-variance matrix of $\left(e_{1}^{i}, x(1)\right)$ is bounded since the covariance matrix of $\left(w_{1}^{k}, u_{1}^{k}, x(1)\right)$ is bounded. The integration and change of variables are need just to show that $p_{e_{1}^{i}, x(1)}$ is bounded.
} 
and define the estimate $\hat{\mathbf{x}}(k)=A_{u}^{-k} \mathbf{x}_{e}(k)$. Since $\mathbf{x}_{u}(k)=\mathbf{x}_{e}(k)+A_{u}^{k} \mathbf{x}_{u}(1)=A_{u}^{k}\left(\hat{\mathbf{x}}(k)-\mathbf{x}_{u}(1)\right)$, we know that:

$$
k \log \left(\left|\operatorname{det}\left(A_{u} A_{u}^{T}\right)\right|\right)+\log \left(\operatorname{det}\left(R_{\mathbf{x}_{\text {error }}}(k)\right)\right)=\log \left(\operatorname{det}\left(R_{\mathbf{x}_{u}}(k, k)\right)\right)<\beta<\infty
$$

where $\mathbf{x}_{\text {error }}(k)=\hat{\mathbf{x}}(k)-\mathbf{x}_{u}(1)$. Since $\hat{x}(k)$ is a function of $e_{1}^{k}$, we have that:

$$
\begin{array}{r}
I\left(\mathbf{x}(1) ; \mathbf{e}_{1}^{k}\right) \geq I\left(\mathbf{x}_{u}(1) ; \mathbf{e}_{1}^{k}\right) \geq h\left(\mathbf{x}_{u}(1)\right)-h\left(\mathbf{x}_{u}(1) \mid \mathbf{e}_{1}^{k}\right)=h\left(\mathbf{x}_{u}(1)\right)-h\left(\mathbf{x}_{u}(1)-\hat{\mathbf{x}}(k) \mid \mathbf{e}_{1}^{k}\right) \geq \\
h\left(\mathbf{x}_{u}(1)\right)-h\left(\hat{\mathbf{x}}(k)-\mathbf{x}_{u}(1)\right)
\end{array}
$$

where we have used (P3), (3), lemma 3.5 of appendix III and (P6).

But, from (P7) we know that $\lim _{\sup _{k \rightarrow \infty}} \frac{h\left(\hat{\mathbf{x}}(k)-\mathbf{x}_{u}(1)\right)}{k} \leq \lim \sup _{k \rightarrow \infty} \frac{\log \left(\operatorname{det}\left(R_{\mathbf{x}_{\text {error }}}(k)\right)\right)}{2 k}$. As a consequence, we can use (21) to get:

$$
\limsup _{k \rightarrow \infty} \frac{h\left(\hat{\mathbf{x}}(k)-\mathbf{x}_{u}(1)\right)}{k} \leq-\log \left(\left|\operatorname{det}\left(A_{u}\right)\right|\right)
$$

The proof follows by direct substitution $\square$.

Using the results in the previous lemmas, we derive theorem 3.3. It states that causality and stability imply that the log-sum of the eigenvalues of $\Sigma\left(\mathbf{e}_{1}^{k}\right)$ are, in the limit, lower bounded by the unstable eigenvalues of the plant.

Theorem 3.3: (Causality fundamental limitation) Let $\mathbf{x}(k)$ be the solution of the state-space equation (8). If the plant is stabilized, i.e., $\sup _{k} \mathcal{E}\left[\mathbf{x}^{T}(k) \mathbf{x}(k)\right]<\infty$ holds then the following is satisfied:

$$
\liminf _{k \rightarrow \infty}\left(L_{-}\left(\mathbf{e}_{1}^{k}\right)+L_{+}\left(\mathbf{e}_{1}^{k}\right)\right) \geq 2 \sum_{i} \max \left\{0, \log \left(\left|\lambda_{i}(A)\right|\right)\right\}
$$

Proof: From lemmas 3.1 and 3.2 we know that:

$$
\liminf _{k \rightarrow \infty} \frac{h\left(\mathbf{e}_{1}^{k}\right)}{k}-h(\mathbf{w}(1)) \geq \liminf _{k \rightarrow \infty} \frac{I\left(\mathbf{e}_{1}^{k} ; \mathbf{x}(1)\right)}{k} \geq \sum_{i} \max \left\{0, \log \left(\left|\lambda_{i}(A)\right|\right)\right\}
$$

Using the fact (P7), we conclude that $h\left(\mathbf{e}_{1}^{k}\right)-k h(\mathbf{w}(1)) \leq \frac{1}{2} \log \left(\operatorname{det}\left(\Sigma\left(\mathbf{e}_{1}^{k}\right)\right)\right)$ which, together with (25), leads to the final result

\section{A. Deriving Bode's Integral Formula}

Under stationarity assumptions, theorem 3.3 is at the base of the Bode-integral formula. A precise description of such property is in the subsequent Corollary. 
Corollary 3.4: Let $\mathrm{x}(k)$ be the solution of the state-space equation (8). If the plant is stabilized, i.e., $\sup _{k} \mathcal{E}\left[\mathbf{x}^{T}(k) \mathbf{x}(k)\right]<\infty$ holds and $\mathbf{e}$ is a stationary process, where $0<m<\hat{F}_{e}(\omega)<M<$ $\infty$ is Lebesgue integrable, then the following is satisfied:

$$
\frac{1}{2 \pi} \int_{-\pi}^{\pi} \log (S(\omega)) d \omega \geq \sum_{i} \max \left\{0, \log \left(\left|\lambda_{i}(A)\right|\right)\right\}
$$

where $S(\omega)=\sqrt{\hat{F}_{e}(\omega)}=\sqrt{\frac{\hat{F}_{\tilde{\tilde{e}}}(\omega)}{\left|G\left(e^{j \omega}\right)\right|^{2}}}$. The processes $\tilde{\mathbf{e}}$ and $\mathbf{d}$ are the ones depicted in Fig 1 .

Proof: From theorem 2.2, of appendix II, we have that:

$$
\lim _{k \rightarrow \infty} L_{-}\left(\mathbf{e}_{1}^{k}\right)+L_{+}\left(\mathbf{e}_{1}^{k}\right)=\frac{1}{2 \pi} \int_{-\pi}^{\pi} \log \left(\hat{F}_{e}(\omega)\right) d \omega
$$

The proof follows by means of (27) and Theorem 3.3 $\square$

\section{Fundamental Limitations CReated by Finite CApacity FeEdback}

In this section, we examine the fundamental limitations, in the eigenvalues of ${ }^{9} \Sigma\left(\mathbf{e}_{g(k)}^{k}\right)$, that originate from the constraint $\bar{I}_{\infty}(\mathbf{v} \rightarrow \mathbf{z}) \leq C_{\text {channel }}$. The main inequality, involving the channel directed information rate and the eigenvalues of $A$, is given in theorem 4.3.

Sub-sequentially, we provide a lemma which unveils how the information flux is allocated in the feedback loop. We identify that the directed information rate in the channel must account for two terms. The first is due to the stabilization information and is given by $I\left(\mathbf{x}(1) ; \mathbf{e}_{1}^{k}\right)$; while the second represents the interaction between the control signal and the disturbance and is quantified by $I\left(\mathbf{u}_{1}^{k} ; \mathbf{w}_{1}^{k}\right)$. The lemma holds if $\mathbf{w}$ is i.i.d. but not necessarily Gaussian ${ }^{10}$.

Lemma 4.1: (Fundamental Lemma of the Information Flux) If $\mathbf{x}(k)$ is the solution of the state-space equation (8) then the following holds:

$$
\bar{I}_{\infty}(\mathbf{v} \rightarrow \mathbf{z}) \geq \liminf _{k \rightarrow \infty} \frac{1}{k} I\left(\mathbf{x}(1) ; \mathbf{e}_{1}^{k}\right)+\bar{I}_{\infty}(\mathbf{u} ; \mathbf{w})
$$

Proof: We start by using (P2) to write $I\left(\left(\mathbf{x}(1), \mathbf{w}_{1}^{k}\right) ; \mathbf{u}_{1}^{k}\right)=I\left(\mathbf{x}(1) ; \mathbf{u}_{1}^{k} \mid \mathbf{w}_{1}^{k}\right)+I\left(\mathbf{u}_{1}^{k} ; \mathbf{w}_{1}^{k}\right)$ which can be rewritten as:

$$
I\left(\left(\mathbf{x}(1), \mathbf{w}_{1}^{k}\right) ; \mathbf{u}_{1}^{k}\right)=I\left(\mathbf{x}(1) ; \mathbf{e}_{1}^{k} \mid \mathbf{w}_{1}^{k}\right)+I\left(\mathbf{u}_{1}^{k} ; \mathbf{w}_{1}^{k}\right)
$$

\footnotetext{
${ }^{9}$ We investigate the eigenvalues of $\Sigma\left(\mathbf{e}_{g(k)}^{k}\right)$, for arbitrary $g$ satisfying $\lim _{k \rightarrow \infty} \frac{g(k)}{k}=0$. Such generalization allows the derivation of an integral formula for exponentially asymptotic stationary processes. More details are provided in section V-A

${ }^{10}$ Assumption (A1) further requires Gaussianity. Such requisite leads to a lower-bound for $I\left(\mathbf{u}_{1}^{k} ; \mathbf{w}_{1}^{k}\right)$, which depends explicitly on the covariance structure of e (see Lemma 4.2).
} 
where we used (P5) to establish that $I\left(\mathbf{x}(1) ; \mathbf{u}_{1}^{k} \mid \mathbf{w}_{1}^{k}\right)=I\left(\mathbf{x}(1) ; \mathbf{e}_{1}^{k} \mid \mathbf{w}_{1}^{k}\right)$. On the other hand, using (P2) we get

$$
I\left(\mathbf{x}(1) ; \mathbf{e}_{1}^{k} \mid \mathbf{w}_{1}^{k}\right)=I\left(\mathbf{x}(1) ; \mathbf{e}_{1}^{k}\right)-I\left(\mathbf{x}(1) ; \mathbf{w}_{1}^{k}\right)+I\left(\mathbf{x}(1) ; \mathbf{w}_{1}^{k} \mid \mathbf{e}_{1}^{k}\right)
$$

Since $\mathbf{w}$ is independent from $\mathbf{x}(1)$, the second term, on the right-hand side of (30), vanishes and we resort to (P1) to get $I\left(\mathbf{x}(1) ; \mathbf{e}_{1}^{k} \mid \mathbf{w}_{1}^{k}\right) \geq I\left(\mathbf{x}(1) ; \mathbf{e}_{1}^{k}\right)$. Consequently, we substitute the aforementioned inequality in (29) and obtain the following:

$$
I\left(\left(\mathbf{x}(1), \mathbf{w}_{1}^{k}\right) ; \mathbf{u}_{1}^{k}\right) \geq I\left(\mathbf{x}(1) ; \mathbf{e}_{1}^{k}\right)+I\left(\mathbf{u}_{1}^{k} ; \mathbf{w}_{1}^{k}\right)
$$

The final inequality follows from (31) and the Theorem 2.1 of appendix II.

The following lemma suggests that attenuation can happen only if the channel conveys information about the disturbance.

Lemma 4.2: The following holds:

$$
\frac{1}{k-k_{0}+1} I\left(\mathbf{u}_{k_{0}}^{k} ; \mathbf{w}_{k_{0}}^{k}\right) \geq-\frac{1}{2} L_{-}\left(\mathbf{e}_{k_{0}}^{k}\right)
$$

Proof: Let the following be the singular value decomposition of $\Sigma\left(\mathbf{e}_{k_{0}}^{k}\right)$ :

$$
\Sigma\left(\mathbf{e}_{k_{0}}^{k}\right)=\left[\begin{array}{l}
V_{+} \\
V_{-}
\end{array}\right]^{T}\left[\begin{array}{cc}
\Lambda_{+} & 0 \\
0 & \Lambda_{-}
\end{array}\right]\left[\begin{array}{l}
V_{+} \\
V_{-}
\end{array}\right]
$$

where $\left[\Lambda_{-}\right]_{i i}<1$ and $\left[\Lambda_{+}\right]_{i i} \geq 1$.

Consequently, we establish the following relation ${ }^{11}$ :

$$
I\left(\mathbf{w}_{k_{0}}^{k} ; \mathbf{u}_{k_{0}}^{k}\right) \geq I\left(V_{-} \mathbf{w}_{k_{0}}^{k} ; V_{-} \mathbf{u}_{k_{0}}^{k}\right)=h\left(V_{-} \mathbf{w}_{k_{0}}^{k}\right)-h\left(V_{-} \mathbf{w}_{k_{0}}^{k} \mid V_{-} \mathbf{u}_{k_{0}}^{k}\right) \geq h\left(V_{-} \mathbf{w}_{k_{0}}^{k}\right)-h\left(V_{-} \mathbf{e}_{k_{0}}^{k}\right)
$$

where we have used (P3), (3), lemma 3.4 of appendix III and (P6). Moreover, since w is i.i.d, $h(\mathbf{w}(k))=\log (2 \pi e)$ and $V$ is unitary, we use (P7) to derive:

$$
h\left(V_{-} \mathbf{w}_{k_{0}}^{k}\right)-h\left(V_{-} \mathbf{e}_{k_{0}}^{k}\right) \geq-\frac{1}{2} \log \left(\operatorname{det}\left(V_{-} \Sigma\left(\mathbf{e}_{k_{0}}^{k}\right) V_{-}^{T}\right)\right)=-\frac{k-k_{0}+1}{2} L_{-}\left(\mathbf{e}_{k_{0}}^{k}\right)
$$

\footnotetext{
${ }^{11}$ Notice that we have used an abuse of notation in equation (34). We write $V_{-} \mathbf{e}_{k_{0}}^{k}$ to indicate the random variable whose realizations are computed as $V_{-}\left[\begin{array}{c}e(k) \\ \vdots \\ e\left(k_{0}\right)\end{array}\right]$
} 
Subsequently, we provide the theorem which states the main inequality in the paper. It reflects a trade-off between disturbance attenuation, as measured by $L_{-}\left(\mathbf{e}_{g(k)}^{k}\right)$, and the directed information rate through the channel, expressed by $\bar{I}_{\infty}(\mathbf{v} \rightarrow \mathbf{z})$.

Theorem 4.3: (Main theorem) Let $\mathbf{x}(k)$ be the solution of the state-space equation (8) and $g: \mathbb{N}_{+} \rightarrow \mathbb{N}_{+}$be an arbitrary function satisfying

$$
\lim _{k \rightarrow \infty} \frac{g(k)}{k}=0
$$

If the plant is stabilized, i.e., $\sup _{k} \mathcal{E}\left[\mathbf{x}^{T}(k) \mathbf{x}(k)\right]<\infty$ then the following is satisfied:

$$
\bar{I}_{\infty}(\mathbf{v} \rightarrow \mathbf{z})-\sum_{i} \max \left\{0, \log \left(\left|\lambda_{i}(A)\right|\right)\right\} \geq-\frac{1}{2} \liminf _{k \rightarrow \infty} L_{-}\left(\mathbf{e}_{g(k)}^{k}\right)
$$

Proof: We begin by using (P3) to arrive at the following fact:

$$
\bar{I}_{\infty}(\mathbf{u} ; \mathbf{w}) \geq \limsup _{k \rightarrow \infty} \frac{1}{k-g(k)+1} I\left(\mathbf{u}_{g(k)}^{k} ; \mathbf{w}_{g(k)}^{k}\right)
$$

The proof follows by substituting the results of lemmas 3.2 and 4.2 into lemma 4.1.

The corollary bellow is an immediate consequence of theorem 4.3 and shows that if $C_{\text {channel }}$ is too close to the critical stabilization rate, given by $\sum_{i} \max \left\{0, \log \left(\left|\lambda_{i}(A)\right|\right)\right\}$, then disturbance rejection is not possible.

Corollary 4.4: Let $\mathbf{x}(k)$ be the solution of the state-space equation (8) and $g(k)$ be a function satisfying

$$
\lim _{k \rightarrow \infty} \frac{g(k)}{k}=0
$$

If the plant is stabilized, i.e., $\sup _{k} \mathcal{E}\left[\mathbf{x}^{T}(k) \mathbf{x}(k)\right]<\infty$ then the following is satisfied:

$$
\frac{1}{2} \liminf _{k \rightarrow \infty} L_{-}\left(\mathbf{e}_{g(k)}^{k}\right)+C_{\text {channel }} \geq \sum_{i} \max \left\{0, \log \left(\left|\lambda_{i}(A)\right|\right)\right\}
$$

Proof: Follows from theorem 4.3 and the fact that $I\left(\mathbf{v}_{1}^{k} \rightarrow \mathbf{z}_{1}^{k}\right) \leq C_{\text {channel }}$.

\section{A. An Integral Formula under Stationarity Assumptions}

Under stationarity assumptions, the condition in theorem 4.3 can be expressed by means of an integral formula.

Corollary 4.5: Let $\mathbf{x}(k)$ be the solution of the state-space equation (8). If the plant is stabilized, i.e., $\sup _{k} \mathcal{E}\left[\mathbf{x}^{T}(k) \mathbf{x}(k)\right]<\infty$ and e is stationary, where $0<m<\hat{F}_{e}(\omega)<M<\infty$ is Lebesgue integrable, then the following is satisfied:

$$
\frac{1}{2 \pi} \int_{-\pi}^{\pi}[\log S(\omega)]_{-} d \omega+\bar{I}_{\infty}(\mathbf{v} \rightarrow \mathbf{z}) \geq \sum_{i} \max \left\{0, \log \left(\left|\lambda_{i}(A)\right|\right)\right\}
$$




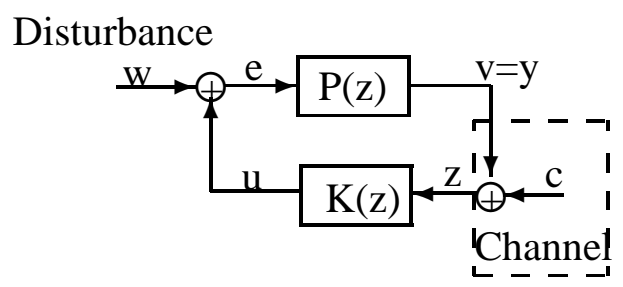

Fig. 3. General structure for a linear feedback loop with disturbances and noisy measurements.

where $S(\omega)=\sqrt{\hat{F}_{e}(\omega)}=\sqrt{\frac{\hat{F}_{\tilde{\tilde{e}}}(\omega)}{\hat{F}_{\mathbf{d}}(\omega)}}=\sqrt{\frac{\hat{F}_{\tilde{\tilde{e}}}(\omega)}{\left|G\left(e^{j \omega}\right)\right|^{2}}}$. The processes $\tilde{\mathbf{e}}$ and $\mathbf{d}$ are the ones depicted in Fig 1.

Proof: By means of a direct application of the theorem 2.2 of appendix II, we find that:

$$
\lim _{k \rightarrow \infty} L_{-}\left(\mathbf{e}_{1}^{k}\right)=\frac{1}{2 \pi} \int_{-\pi}^{\pi}\left[\log \left(\hat{F}_{e}(\omega)\right)\right]_{-} d \omega
$$

The result follows by direct substitution of (40) in (36). $\square$ From Lemma 5.1, we conclude that Corollary 4.5 remains valid if, instead of stationarity, we only require exponential asymptotic stationarity.

\section{Case Study: The Linear Gaussian Case}

Consider the linear feedback loop of Fig 3 and that the blocks and signals represented satisfy:

- (E1) $P(z)$ is a strictly proper linear and time-invariant plant of order $n_{p}$.

- (E2) $K(z)$ is a proper linear and time invariant system of order $n_{K}$

- (E3) $\mathbf{w}$ is a zero mean Gaussian i.i.d. and unit variance random process. The process $\mathbf{c}$ is i.i.d. and Gaussian, with variance $\sigma_{c}^{2}$.

- (E4) $\mathbf{x}(1)$ is also zero mean Gaussian.

- (E5) the initial state of $K$ is taken as $x_{K}(1)=0$

- (E6) the feedback loop is stable.

The example of Fig 3 is a particular instance of the scheme of Fig 2. All assumptions stated in section II-A are satisfied, in particular (A7) and (A8). Since we assume that $K(z)$ has zero initial state then (A7) is satisfied with $\alpha=0$ and (A8) is also immediately true.

In the following sub-sections, we discuss a numerical computation which suggests that the following inequality (from the main Theorem 4.3) is not conservative:

$$
\bar{I}_{\infty}(\mathbf{v} \rightarrow \mathbf{z})-\sum_{i} \max \left\{0, \log \left(\left|\lambda_{i}(A)\right|\right)\right\} \geq-\frac{1}{2} \liminf _{k \rightarrow \infty} L_{-}\left(\mathbf{e}_{g(k)}^{k}\right)
$$




\section{A. Preliminary Results: Extension to the Non-Stationary Case}

The aim of this subsection is to derive computable upper and lower bounds for the inequality (41). The subsequent Lemmas comprise integral formulas for the Gaussian asymptotic stationary case. The final inequality is presented in Corollary 5.4, where the upper and lower bounds are easily computable through integrals. Consequently, we can obtain the numerical results of section V-B and test for the tightness of the inequality (41).

We emphasize that, all the quantities in the statements and in the proofs of this subsection, refer to the example of Fig 3. As such, we assume that they comply with (E1)-(E6).

Lemma 5.1: Let $\overrightarrow{\mathrm{e}}$ be a stationary stochastic process with auto-covariance $R_{\vec{e}}(\tau)$ and a power spectral density $\hat{F}_{\vec{e}}(\omega)$ which is a Lebesgue integrable function satisfying $0<m<\hat{F}_{\vec{e}}(\omega)<$ $M<\infty$. Consider also a, not necessarily stationary, stochastic process e with auto-covariance $R_{e}\left(k_{0}, k_{1}\right)$. If the condition below holds:

$$
\exists \beta>0, \exists \gamma \in(0,1) \text { such that } \forall k_{0}, \forall \tau>0,\left|R_{e}\left(k_{0}, k_{0}+\tau\right)-R_{\vec{e}}(\tau)\right|<\beta \gamma^{k_{0}}
$$

then the following integral equation is satisfied:

$$
\liminf _{k \rightarrow \infty} L_{-}\left(\mathbf{e}_{k}^{k^{2}}\right)=\frac{1}{2 \pi} \int_{-\pi}^{\pi}\left[\log \left(\hat{F}_{\vec{e}}(\omega)\right)\right]_{-} d \omega
$$

Proof: We follow the same steps of the proof of lemma 4.2 and get the following diagonalization of $\Sigma\left(\overrightarrow{\mathbf{e}}_{k}^{k^{2}}\right)$ :

$$
\Sigma\left(\overrightarrow{\mathbf{e}}_{k}^{k^{2}}\right)=\left[\begin{array}{c}
V_{k,+} \\
V_{k,-}
\end{array}\right]^{T}\left[\begin{array}{cc}
\Lambda_{k,+} & 0 \\
0 & \Lambda_{k,-}
\end{array}\right]\left[\begin{array}{l}
V_{k,+} \\
V_{k,-}
\end{array}\right]
$$

where $\left[\Lambda_{k,-}\right]_{i i}<1$ and $\left[\Lambda_{k,+}\right]_{i i} \geq 1$. Now, assumption (42) and Theorem 2.2 of Appendix II, guarantee that:

$$
\begin{aligned}
& \liminf _{k \rightarrow \infty} L_{-}\left(\mathbf{e}_{k}^{k^{2}}\right)=\liminf _{k \rightarrow \infty} \frac{1}{k^{2}-k+1} \log \left(\operatorname{det}\left(V_{k,-} \Sigma\left(\mathbf{e}_{k}^{k^{2}}\right) V_{k,-}^{T}\right)\right) \underset{(*)}{=} \\
& \liminf _{k \rightarrow \infty} \frac{1}{k^{2}-k+1} \log \left(\operatorname{det}\left(V_{k,-} \Sigma\left(\overrightarrow{\mathbf{e}}_{k}^{k^{2}}\right) V_{k,-}^{T}\right)\right)=\lim _{k \rightarrow \infty} L_{-}\left(\overrightarrow{\mathbf{e}}_{k}^{k^{2}}\right)=\frac{1}{2 \pi} \int_{-\pi}^{\pi}\left[\log \left(\hat{F}_{\vec{e}}(\omega)\right)\right]_{-} d \omega
\end{aligned}
$$

The proof is complete, once we provide more detail on the validity of the equality marked with $\left.{ }^{*}\right)$ in (45). From (42) and the fact that $\left[\Lambda_{k,-}\right]_{i i} \geq m$, we know that:

$$
\lim _{k \rightarrow \infty} \varrho\left(\Delta_{k, e}\right)=0
$$


where $\varrho\left(\Delta_{k, e}\right)=\max _{i} \sum_{j}\left|\left[\Delta_{k, e}\right]_{i, j}\right|$ and $\Delta_{k, e}$ is a matrix satisfying:

$$
V_{k,-} \Sigma\left(\mathbf{e}_{k}^{k^{2}}\right) V_{k,-}^{T}=\underbrace{V_{k,-} \Sigma\left(\overrightarrow{\mathbf{e}}_{k}^{k^{2}}\right) V_{k,-}^{T}}_{\Lambda_{k,-}}+V_{k,-}\left(\Sigma\left(\mathbf{e}_{k}^{k^{2}}\right)-\Sigma\left(\overrightarrow{\mathbf{e}}_{k}^{k^{2}}\right)\right) V_{k,-}^{T}=\Lambda_{k,-}\left(I+\Delta_{k, e}\right)
$$

From Gershgorin's circle Theorem we infer that $\lambda_{i}\left(I+\Delta_{k, e}\right) \in\left[1-\varrho\left(\Delta_{k, e}\right), 1+\varrho\left(\Delta_{k, e}\right)\right]$, which concludes the proof.

Lemma 5.2: Let $\mathbf{v}$ and $\mathbf{z}$ be the stochastic processes represented in Figure 3, i.e., $\mathbf{z}=\mathbf{v}+\mathbf{c}$. Consider also that $\overrightarrow{\mathbf{z}}$ is a stationary stochastic process with auto-covariance $R_{\vec{z}}(\tau)$ and a power spectral density $\hat{F}_{\vec{z}}(\omega)$ which is a Lebesgue integrable function satisfying $0<m<\hat{F}_{\vec{z}}(\omega)<$ $M<\infty$. If the condition below holds:

$$
\exists \beta>0, \gamma \in(0,1) \text { such that } \forall k_{0}, \tau>0,\left|R_{z}\left(k_{0}, k_{0}+\tau\right)-R_{\vec{z}}(\tau)\right|<\beta \gamma^{k_{0}}
$$

then the following is satisfied:

$$
\frac{1}{4 \pi} \int_{-\pi}^{\pi} \log \left(\frac{\hat{F}_{\vec{z}}(\omega)}{\sigma_{c}^{2}}\right) d \omega \geq \bar{I}_{\infty}(\mathbf{v} \rightarrow \mathbf{z})
$$

Proof: Choose arbitrary $\nu \in \mathbb{N}_{+}$. We start by noticing that:

$$
\begin{aligned}
\limsup _{k \rightarrow \infty} h\left(\mathbf{z}(k) \mid \mathbf{z}_{k-\nu+1}^{k-1}\right)-h(\mathbf{c}(k)) \geq & \\
& \limsup _{k \rightarrow \infty} h\left(\mathbf{z}(k) \mid \mathbf{z}_{1}^{k-1}\right)-h(\mathbf{c}(k))=\limsup _{k \rightarrow \infty} I\left(\mathbf{z}(k) ; \mathbf{v}_{1}^{k} \mid \mathbf{z}_{1}^{k-1}\right) \geq \bar{I}_{\infty}(\mathbf{v} \rightarrow \mathbf{z})
\end{aligned}
$$

Now, notice that assumption (48), guarantees that:

$$
\lim _{k \rightarrow \infty} h\left(\mathbf{z}(k) \mid \mathbf{z}_{k-\nu+1}^{k-1}\right)=\lim _{k \rightarrow \infty} h\left(\overrightarrow{\mathbf{z}}(k) \mid \overrightarrow{\mathbf{z}}_{k-\nu+1}^{k-1}\right) \underset{\text { stationarity }}{=} h\left(\overrightarrow{\mathbf{z}}(\nu) \mid \overrightarrow{\mathbf{z}}_{1}^{\nu-1}\right)
$$

which, from (50), implies:

$$
h\left(\overrightarrow{\mathbf{z}}(\nu) \mid \overrightarrow{\mathbf{z}}_{1}^{\nu-1}\right)-h(\mathbf{c}(1)) \geq \bar{I}_{\infty}(\mathbf{v} \rightarrow \mathbf{z})
$$

Since $\nu$ was arbitrary, we can use (52) and Theorem 2.2, from Appendix II, to state that:

$$
\bar{I}_{\infty}(\mathbf{v} \rightarrow \mathbf{z}) \leq \lim _{\nu \rightarrow \infty} h\left(\overrightarrow{\mathbf{z}}(\nu) \mid \overrightarrow{\mathbf{z}}_{1}^{\nu-1}\right)-h(\mathbf{c}(1))=\frac{1}{4 \pi} \int_{-\pi}^{\pi} \log \left(\frac{\hat{F}_{\vec{z}}(\omega)}{\sigma_{c}^{2}}\right) d \omega
$$

Theorem 5.3: If the feedback system of Fig 3 is stable then the following holds:

$$
\begin{aligned}
& \frac{1}{4 \pi} \int_{-\pi}^{\pi} \log \left(\frac{\hat{F}_{\vec{z}}(\omega)}{\sigma_{c}^{2}}\right) d \omega-\sum_{i} \max \left\{0, \log \left(\left|\lambda_{i}(A)\right|\right)\right\} \\
& \bar{I}_{\infty}(\mathbf{v} \rightarrow \mathbf{z})-\sum_{i} \max \left\{0, \log \left(\left|\lambda_{i}(A)\right|\right)\right\} \geq-\frac{1}{2} \liminf _{k \rightarrow \infty} L_{-}\left(\mathbf{e}_{g(k)}^{k}\right)= \\
& \quad-\frac{1}{4 \pi} \int_{-\pi}^{\pi}\left[\log \left(\hat{F}_{\vec{e}}(\omega)\right)\right]_{-} d \omega
\end{aligned}
$$


Proof: Since the system is stable, the exponential asymptotic stationarity conditions of Lemmas 5.1 and 5.2 are satisfied. The result follows from these Lemmas and the main Theorem 4.3.

The following Corollary, specializes Theorem 5.3 to the feed-back loop of Fig 3:

Corollary 5.4: If the feedback system of Fig 3 is stable then the following holds:

$$
\begin{aligned}
& \frac{1}{4 \pi} \int_{-\pi}^{\pi} \log \left(1+\frac{\left|P\left(e^{j \omega}\right)\right|^{2}}{\sigma_{c}^{2}}\right) d \omega \\
& \bar{I}_{\infty}(\mathbf{v} \rightarrow \mathbf{z})-\sum_{i} \max \left\{0, \log \left(\left|\lambda_{i}(A)\right|\right)\right\} \geq-\frac{1}{2} \liminf _{k \rightarrow \infty} L_{-}\left(\mathbf{e}_{g(k)}^{k}\right)= \\
& \quad-\frac{1}{4 \pi} \int_{-\pi}^{\pi}\left[\log \left(\frac{1+\sigma_{c}^{2}\left|K\left(e^{j \omega}\right)\right|^{2}}{\left|1+P\left(e^{j \omega}\right) K\left(e^{j \omega}\right)\right|^{2}}\right)\right]_{-} d \omega
\end{aligned}
$$

Proof: We start by computing the power spectral density $\hat{F}_{\vec{z}}(\omega)$ to obtain:

$$
\frac{\hat{F}_{\vec{z}}(\omega)}{\sigma_{c}^{2}}=\frac{1}{\left|1+P\left(e^{j \omega}\right) K\left(e^{j \omega}\right)\right|^{2}}\left(1+\frac{\left|P\left(e^{j \omega}\right)\right|^{2}}{\sigma_{c}^{2}}\right)
$$

But, from the residue theorem, we use (56) to show that:

$$
\frac{1}{4 \pi} \int_{-\pi}^{\pi} \log \left(\frac{\hat{F}_{\vec{z}}(\omega)}{\sigma_{c}^{2}}\right) d \omega=\frac{1}{4 \pi} \int_{-\pi}^{\pi} \log \left(1+\frac{\left|P\left(e^{j \omega}\right)\right|^{2}}{\sigma_{c}^{2}}\right) d \omega+\sum_{i} \max \left\{0, \log \left(\left|\lambda_{i}(A)\right|\right)\right\}
$$

The power spectral density of $\hat{F}_{\vec{e}}(\omega)$ leads to:

$$
\frac{\hat{F}_{\vec{e}}(\omega)}{\sigma_{c}^{2}}=\frac{1+\sigma_{c}^{2}\left|K\left(e^{j \omega}\right)\right|^{2}}{\left|1+P\left(e^{j \omega}\right) K\left(e^{j \omega}\right)\right|^{2}}
$$

The proof is concluded by direct substitution of (57) and (58) in Theorem 5.3.

\section{B. Numerical results}

From Corollary 5.4, we infer that an indication for the tightness of the inequality (41) is that the following lower-bound and upper-bound are close:

$$
\begin{gathered}
u b\left(\sigma_{c}^{2}\right)=\frac{1}{4 \pi} \int_{-\pi}^{\pi} \log \left(1+\frac{\left|P\left(e^{j \omega}\right)\right|^{2}}{\sigma_{c}^{2}}\right) d \omega \\
l b\left(\sigma_{c}^{2}\right)=-\frac{1}{4 \pi} \int_{-\pi}^{\pi}\left[\log \left(\frac{1+\sigma_{c}^{2}\left|K\left(e^{j \omega}\right)\right|^{2}}{\left|1+P\left(e^{j \omega}\right) K\left(e^{j \omega}\right)\right|^{2}}\right)\right]_{-} d \omega
\end{gathered}
$$

In Figure 4, we depict the numerical results for the following $P$ and $K$ :

$$
\begin{gathered}
P(z)=\frac{z^{-1}}{\left(1-1.5 z^{-1}\right)^{10}} \\
K(z)=z-\frac{1}{P(z)}
\end{gathered}
$$




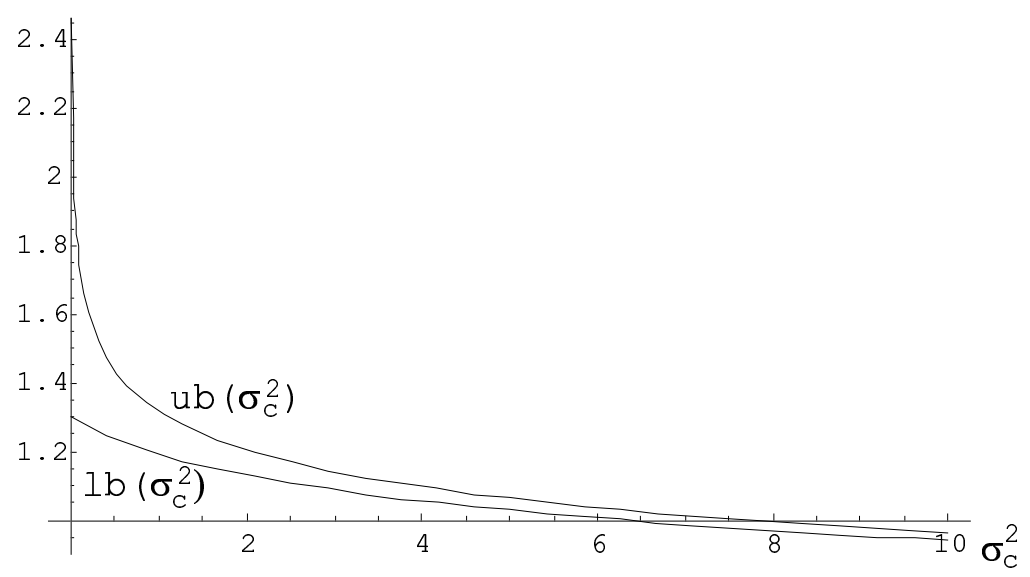

Fig. 4. Plot of the upper-bound and lower-bound, computed as a function of $\sigma_{c}^{2}$.

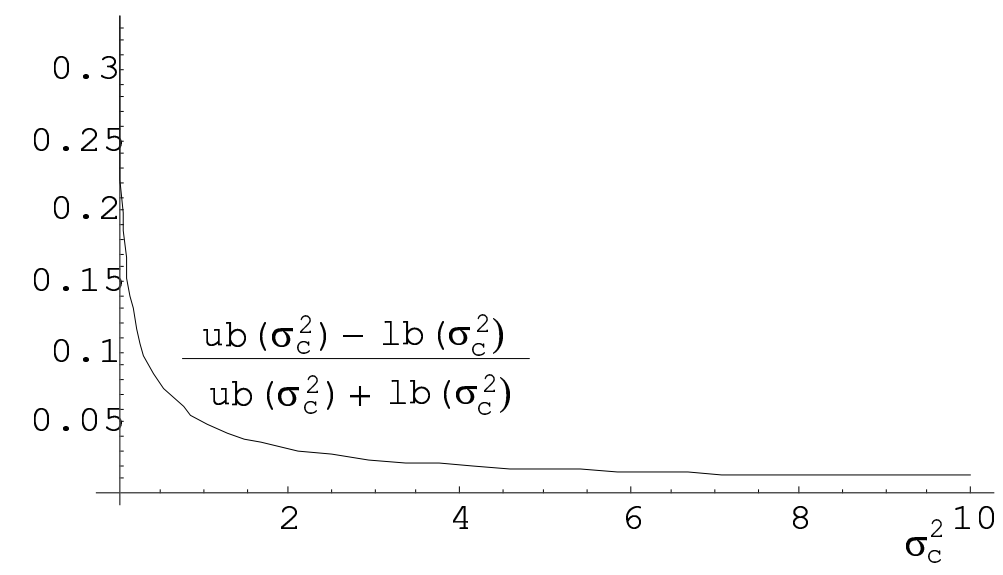

Fig. 5. Plot of the relative difference between the upper-bound and lower-bound, computed as a function of $\sigma_{c}^{2}$.

Notice that $K$ is the dead-bit controller. We emphasize that the choice of the multiple pole of $P(z)$ was arbitrary. We have tried other values and the bounds behaved in a similar way.

By inspection, one can argue that the bounds get more accurate for increasing values of $\sigma_{c}^{2}$ (see Fig 5). Moreover, we have verified empirically that such relative accuracy can be made arbitrarily small by considering $P(z)=\frac{z^{-1}}{\left(1-1.5 z^{-1}\right)^{n}}$, with $n$ arbitrarily large and $K(z)=z-\frac{1}{P(z)}$.

\section{CONCLUSIONS}

Through an information theoretic viewpoint, this paper addresses two fundamental trade-offs of performance for the feedback loop represented in Figure 1. 
As a first step, we show that causality leads to the extension of Bode's Fundamental limitation and the water-bed effect. Under stationarity assumptions, the aforementioned extension is identical to the standard integral formula.

Secondly, we provide a trade-off between disturbance attenuation and the directed information rate at the channel $\bar{I}_{\infty}(\mathbf{v} \rightarrow \mathbf{z})$. Previous results have established that $\bar{I}_{\infty}(\mathbf{v} \rightarrow \mathbf{z}) \geq$ $\sum_{\text {unstable }} \log \left(\lambda_{i}(A)\right)$ is necessary for stability and our analysis shows that disturbance attenuation is achieved at the expense of the extra rate given by $\bar{I}_{\infty}(\mathbf{v} \rightarrow \mathbf{z})-\sum_{\text {unstable }} \log \left(\lambda_{i}(A)\right)$.

The inequalities presented in the paper unveil how information flows in the loop. We expect that our results will motivate further work in the direction of designing encoders and decoders that meet certain performance specifications.

\section{APPENDIX I}

\section{ASPECTS RELATED TO ASSUMPTIONS (A5), (A7)-(A9)}

\section{A. Further comments on assumption (A5)}

The following upper-bound holds:

$$
\bar{I}_{\infty}(\mathbf{v} \rightarrow \mathbf{z}) \leq C_{\text {channel }}
$$

By means of property (P3), we know that the following is satisfied:

$$
\forall k \geq i, I\left(\mathbf{z}(i) ; \mathbf{v}_{1}^{i} \mid \mathbf{z}_{1}^{i-1}\right) \leq I\left(\mathbf{z}(i) ; \mathbf{v}_{1}^{k} \mid \mathbf{z}_{1}^{i-1}\right) \Longrightarrow \bar{I}_{\infty}(\mathbf{v} \rightarrow \mathbf{z}) \leq \bar{I}_{\infty}(\mathbf{v} ; \mathbf{z}) \leq C_{\text {channel }}
$$

where the last inequality is standard and can be found in [4].

\section{B. About (A7)}

Notice that a synchronous block decoder, with delay $\alpha$, falls into this category. In addition, any dynamic system, of the form $\mathbf{u}(k)=f\left(\mathbf{u}_{k-\alpha}^{k-1}, \mathbf{z}_{k-\alpha}^{k}\right)$, will satisfy (9). We emphasize that this representation does not pressupose a full-information system. For example, if $\mathbf{y}_{\mathbf{c}}(k)$ is the output of an observable n-th order linear and time-invariant system, with input $\mathbf{z}(k)$, then it is possible to represent its input-output behavior in the form $\mathbf{y}_{\mathbf{c}}(k)=f\left(\mathbf{y}_{\mathbf{c}_{k-n}}^{k-1}, \mathbf{z}_{k-n}^{k}\right)$.

\section{Further Remarks About Assumption (A8)}

The following is the assumption (A8), repeated here for convenience:

$$
\lim _{k \rightarrow \infty} \frac{1}{k} I\left(\mathbf{u}_{1}^{\alpha} ;\left(\mathbf{x}(1), \mathbf{w}_{1}^{k}\right) \mid \mathbf{z}_{1}^{k}\right)=0
$$


1) Assumption (A8) when $\mathcal{U}$ is countable: If $\mathcal{U}$ is countable then we can use (P1)-(P2) to conclude that:

$$
I\left(\mathbf{u}_{1}^{\alpha} ; \mathbf{w}_{1}^{k} \mid \mathbf{z}_{1}^{k}\right) \leq H\left(\mathbf{u}_{1}^{\alpha}\right)
$$

As such, if $H\left(\mathbf{u}_{1}^{\alpha}\right)<\infty$ holds then (64) is satisfied. If $\mathcal{U}$ has $\aleph_{U}$ elements, such quantity is upperbounded [4] as $H\left(\mathbf{u}_{1}^{\alpha}\right) \leq \alpha \log \left(\aleph_{U}\right)$. The confinement to finite control alphabets is expected if the channel, itself, is discrete or in the presence of quantizers. Finite $\mathcal{U}$ further encompasses digital controllers, as they constitute dynamic systems evolving on a finite precision algebra. The following proposition is also useful:

Proposition 1.1: Let $\mathcal{U}$ be countable, $\mathbf{u}_{1}^{\alpha} \in \mathbb{D}$ and $p_{\breve{u}(i)} \in \overline{\mathbb{L}}^{1}$ for $i \in\{1, \ldots, \alpha\}$. If $\operatorname{Var}(\mathbf{u}(i))<$ $\infty$ for $i \in\{1, \ldots, \alpha\}$ then the following holds:

$$
\lim _{k \rightarrow \infty} \frac{1}{k} I\left(\mathbf{u}_{1}^{\alpha} ;\left(\mathbf{x}(1), \mathbf{w}_{1}^{k}\right) \mid \mathbf{z}_{1}^{k}\right)=0
$$

Proof: Since $\mathbf{u}_{1}^{\alpha} \in \mathbb{D}$, we can compute $\Delta>0$ as:

$$
\Delta=\inf \{|u-\tilde{u}|: u, \tilde{u} \in \mathcal{U}, u \neq \tilde{u}\}
$$

We start by means of proposition 3.1 of Appendix III, we can use $\operatorname{Var}(\breve{\mathbf{u}}(i))=\operatorname{Var}(\mathbf{u}(i))+\frac{\Delta^{2}}{4}<$ $\infty$ and $p_{u(i)} \in \overline{\mathbb{L}}^{1}$ to reach the following:

$$
h(\breve{\mathbf{u}}(i))<\infty
$$

On the other hand, such integral can be related to $H(\mathbf{u}(i))$ as:

$$
I\left(\mathbf{u}_{1}^{\alpha} ;\left(\mathbf{x}(1), \mathbf{w}_{1}^{k}\right) \mid \mathbf{z}_{1}^{k}\right) \leq H\left(\mathbf{u}_{1}^{\alpha}\right) \leq \sum_{i=1}^{\alpha} H(\mathbf{u}(i))=\sum_{i=1}^{\alpha} h(\breve{\mathbf{u}}(i))-\log (\Delta)<\infty
$$

where we use the fact that $p_{\breve{u}(i)}(\breve{u}(i))=\mathcal{P}(\mathbf{u}(i)=u(i)) \frac{1}{\Delta} p_{s}\left(\frac{\breve{u}(i)-u(i)}{\Delta}\right)$ and $p_{s}(s)=1$ if $s \in$ $(-1 / 2,1 / 2)$ and $p_{s}(s)=0$ otherwise.

\section{Further remarks about assumption (A9)}

We start our comments, about (A9), by saying that we require that $p_{\mathbf{w}_{1}^{k}, \mathbf{x}(1), \breve{u}_{1}^{k}} \in \mathbb{L}^{2 k+1}$, just as a way to compactly guarantee that any $q$ dimensional marginal distribution is in $\overline{\mathbb{L}}^{q}$. This condition is important to ensure that the differential entropy integrals are well defined.

If $\mathcal{U}_{1}^{k}$ is not countable and $p_{\mathbf{w}_{1}^{k}, \mathbf{x}(1), \breve{u}_{1}^{k}}$ is Gaussian then $p_{\mathbf{w}_{1}^{k}, \mathbf{x}(1), \breve{u}_{1}^{k}} \in \mathbb{L}^{2 k+1}$ holds if and only if the covariance matrix of $\left(\mathbf{w}_{1}^{k}, \mathbf{x}(1), \breve{\mathbf{u}}_{1}^{k}\right)$ is positive definite. 


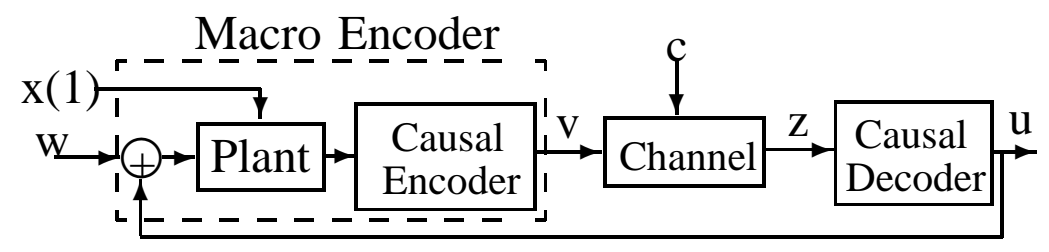

Fig. 6. Casting the feedback loop as a channel in feedback.

We list a few facts of relevance about assumption (A9), for the case where $\mathbf{u}(k)$ is on a countable alphabet:

- Notice that if $\mathcal{U}$ is countable then $\operatorname{Var}(\breve{\mathbf{u}}(k))=\operatorname{Var}(\mathbf{u}(k))+\frac{\Delta^{2}}{4}$.

- It remains to show that there are no measurability problems, provided that $p_{x(1)}$ is measurable. Assume that $\mathrm{x}(1)$ has a bounded and Lebesgue measurable $p_{x(1)}$. For every Borel set $\mathcal{O} \in \mathcal{R}^{k+1}$, we have:

$\exists \beta>0, \mathcal{P}\left(\left(\mathbf{w}_{1}^{k}, \mathbf{x}(1)\right) \in \mathcal{O}, \mathbf{u}_{1}^{k}=u_{1}^{k}\right) \leq \int_{\mathcal{O}} p_{w_{1}^{k}}\left(\gamma_{w_{1}^{k}}\right) p_{x(1)}\left(\gamma_{x(1)}\right) \mu\left(d \gamma_{w_{1}^{k}} \times d \gamma_{x(1)}\right) \leq \beta \operatorname{Vol}(\mathcal{O})$

where we used assumption (A1) to guarantee the existence of a bounded and Lebesgue measurable $p_{w_{1}^{k}}$ and causality to split $p_{w_{1}^{k}, x(1)}=p_{w_{1}^{k}} p_{x(1)}$. As such, from (70) and the Radon-Nikodym theorem (pp.422 [2]), we know that, for each $u_{1}^{k} \in \mathcal{U}^{k}$, there exists a measurable probability density function $p_{\mathbf{w}_{1}^{k}, \mathbf{x}(1) \mid u_{1}^{k}}\left(\cdot, \cdot, u_{1}^{k}\right): \mathbb{R}^{k+1} \rightarrow \mathbb{R}_{\geq 0}$. On the other hand, $p_{\mathbf{w}_{1}^{k}, \mathbf{x}(1), \breve{u}_{1}^{k}}$ is given as:

$$
\begin{gathered}
p_{w_{1}^{k}, x(1), \breve{u}_{1}^{k}}\left(\gamma_{w_{1}^{k}}, \gamma_{x(1)}, \gamma_{\breve{u}_{1}^{k}}\right)=\sum_{u_{1}^{k} \in \mathcal{U}^{k}} p_{\mathbf{w}_{1}^{k}, \mathbf{x}(1) \mid u_{1}^{k}}\left(\gamma_{w_{1}^{k}}, \gamma_{x(1)}, u_{1}^{k}\right) p_{s}\left(\gamma_{\breve{u}_{1}^{k}}-u_{1}^{k}\right) \mathcal{P}\left(\mathbf{u}_{1}^{k}=u_{1}^{k}\right) \\
\mathbf{s}=\breve{\mathbf{u}}_{1}^{k}-\mathbf{u}_{1}^{k}
\end{gathered}
$$

which allows us to infer that $p_{\mathbf{w}_{1}^{k}, \mathbf{x}(1), \breve{u}_{1}^{k}}$ is a countable linear combination of positive Lebesgue measurable functions. Clearly, besides (A1), we only need to assume bounded and Lebesgue measurable $p_{x(1)}$ to guarantee that $p_{\mathbf{w}_{1}^{k}, \mathbf{x}(1), \breve{u}_{1}^{k}}$ is bounded and Lebesgue measurable.

\section{APPENDIX II}

\section{AUXILIARY RESULTS}

The following theorem provides an extension of the directed data processing inequality, originally derived in [20]. Compared to the version in [20], the result presented bellow allows 
encoders and decoders that depend on past inputs indexed by $k<1$. The quantities in the statement of the theorem refer to the scheme depicted in Fig 6.

Theorem 2.1: (Directed Data Processing Inequality, Adaptation of Lemma 4.8.1 of [20]) Let the following assumptions, stated in section II-A and summarized bellow for convenience, hold:

- (A4) The plant is LTI with a state-space representation where $D=0$ (strictly proper)

- (A6) The encoder and decoder are causal operators

- (A7) The decoder satisfies:

$$
\forall k>\alpha, \mathbf{u}_{\alpha+1}^{k}=\tilde{f}_{k}^{d}\left(\mathbf{u}_{1}^{\alpha}, \mathbf{z}_{1}^{k}\right)
$$

for some $\alpha \in \mathbb{N}_{+}$and a sequence of functions $\tilde{f}_{k}^{d}$.

- (A8) The fading memory condition $\lim \sup _{k \rightarrow \infty} \frac{1}{k} I\left(\mathbf{u}_{1}^{\alpha} ;\left(\mathbf{x}(1), \mathbf{w}_{1}^{k}\right) \mid \mathbf{z}_{1}^{k}\right)=0$ holds.

Under the above conditions, the following is true:

$$
\limsup _{k \rightarrow \infty} \frac{1}{k} I\left(\left(\mathbf{x}(1), \mathbf{w}_{1}^{k}\right) ; \mathbf{u}_{1}^{k}\right) \leq \bar{I}_{\infty}(\mathbf{v} \rightarrow \mathbf{z})
$$

Proof: We separate the proof in two parts.

As a first step we show that $I\left(\mathbf{z}_{1}^{k} ;\left(\mathbf{x}(1), \mathbf{w}_{1}^{k}\right)\right) \leq I\left(\mathbf{v}_{1}^{k} \rightarrow \mathbf{z}_{1}^{k}\right)$.

Using (P2) we can write the following equality, for any given $i \in\{1, \ldots, k\}$ :

$$
\begin{array}{r}
I\left(\mathbf{z}(i) ;\left(\mathbf{x}(1), \mathbf{w}_{1}^{i-1}\right) \mid \mathbf{z}_{1}^{i-1}\right)=I\left(\mathbf{z}(i) ; \mathbf{v}_{1}^{i} \mid \mathbf{z}_{1}^{i-1}\right)+I\left(\mathbf{z}(i) ;\left(\mathbf{x}(1), \mathbf{w}_{1}^{i-1}\right) \mid \mathbf{z}_{1}^{i-1}, \mathbf{v}_{1}^{i}\right) \\
-I\left(\mathbf{z}(i) ; \mathbf{v}_{1}^{i} \mid \mathbf{z}_{1}^{i-1}, \mathbf{x}(1), \mathbf{w}_{1}^{i-1}\right)
\end{array}
$$

Now notice that $(\mathrm{P} 2)$ allows us to rewrite:

$$
I\left(\mathbf{z}(i) ;\left(\mathbf{x}(1), \mathbf{w}_{1}^{i-1}\right) \mid \mathbf{z}_{1}^{i-1}, \mathbf{v}_{1}^{i}\right)=I\left(\left(\mathbf{z}_{1}^{i}, \mathbf{v}_{1}^{i}\right) ;\left(\mathbf{x}(1), \mathbf{w}_{1}^{i-1}\right)\right)-I\left(\left(\mathbf{z}_{1}^{i-1}, \mathbf{v}_{1}^{i}\right) ;\left(\mathbf{x}(1), \mathbf{w}_{1}^{i-1}\right)\right)
$$

But, from (P3), we know that

$$
I\left(\left(\mathbf{z}_{1}^{i}, \mathbf{v}_{1}^{i}\right) ;\left(\mathbf{x}(1), \mathbf{w}_{1}^{i-1}\right)\right)=I\left(\left(\mathbf{c}(i), \mathbf{z}_{1}^{i-1}, \mathbf{v}_{1}^{i}\right) ;\left(\mathbf{x}(1), \mathbf{w}_{1}^{i-1}\right)\right)
$$

where we used the fact that, from the definition 1.7 (channel), the following map is invertible:

$$
(z(i), v(i)) \longmapsto(c(i), v(i))
$$

Causality makes $\mathbf{c}(i)$ independent of $\left(\mathbf{z}_{1}^{i-1}, \mathbf{v}_{1}^{i}, \mathbf{x}(1), \mathbf{w}_{1}^{i-1}\right)$, so that (77) implies the following:

$$
I\left(\left(\mathbf{z}_{1}^{i}, \mathbf{v}_{1}^{i}\right) ;\left(\mathbf{x}(1), \mathbf{w}_{1}^{i-1}\right)\right)=I\left(\left(\mathbf{z}_{1}^{i-1}, \mathbf{v}_{1}^{i}\right) ;\left(\mathbf{x}(1), \mathbf{w}_{1}^{i-1}\right)\right)
$$


By making use of (78) and (76) we infer that $I\left(\mathbf{z}(i) ;\left(\mathbf{x}(1), \mathbf{w}_{1}^{i-1}\right) \mid \mathbf{z}_{1}^{i-1}, \mathbf{v}_{1}^{i}\right)=0$. Such fact, together with (P1) and (75), leads to:

$$
I\left(\mathbf{z}(i) ;\left(\mathbf{x}(1), \mathbf{w}_{1}^{i-1}\right) \mid \mathbf{z}_{1}^{i-1}\right) \leq I\left(\mathbf{z}(i) ; \mathbf{v}_{1}^{i} \mid \mathbf{z}_{1}^{i-1}\right)
$$

The first part of the proof is concluded once we notice that, from causality, $\mathbf{w}_{i}^{k}$ is independent of $\left(\mathbf{x}(1), \mathbf{w}_{1}^{i-1}, \mathbf{z}_{1}^{i}\right)$, which implies:

$$
I\left(\mathbf{z}(i) ;\left(\mathbf{x}(1), \mathbf{w}_{1}^{k}\right) \mid \mathbf{z}_{1}^{i-1}\right)=I\left(\mathbf{z}(i) ;\left(\mathbf{x}(1), \mathbf{w}_{1}^{i-1}\right) \mid \mathbf{z}_{1}^{i-1}\right)
$$

so that (79) implies:

$$
I\left(\mathbf{z}_{1}^{k} ;\left(\mathbf{x}(1), \mathbf{w}_{1}^{k}\right)\right)=\sum_{i=1}^{k} I\left(\mathbf{z}(i) ;\left(\mathbf{x}(1), \mathbf{w}_{1}^{i-1}\right) \mid \mathbf{z}_{1}^{i-1}\right) \leq \sum_{i=1}^{k} I\left(\mathbf{z}(i) ; \mathbf{v}_{1}^{i} \mid \mathbf{z}_{1}^{i-1}\right)=I\left(\mathbf{v}_{1}^{k} \rightarrow \mathbf{z}_{1}^{k}\right)
$$

In the second step we prove that $\lim \sup _{k \rightarrow \infty} \frac{1}{k} I\left(\mathbf{u}_{1}^{k} ;\left(\mathbf{x}(1), \mathbf{w}_{1}^{k}\right)\right) \leq \lim \sup _{k \rightarrow \infty} \frac{1}{k} I\left(\mathbf{z}_{1}^{k} ;\left(\mathbf{x}(1), \mathbf{w}_{1}^{k}\right)\right)$.

Once again, we use (P2) to write:

$$
I\left(\mathbf{u}_{1}^{k} ;\left(\mathbf{x}(1), \mathbf{w}_{1}^{k}\right)\right)=I\left(\mathbf{z}_{1}^{k} ;\left(\mathbf{x}(1), \mathbf{w}_{1}^{k}\right)\right)+I\left(\mathbf{u}_{1}^{k} ;\left(\mathbf{x}(1), \mathbf{w}_{1}^{k}\right) \mid \mathbf{z}_{1}^{k}\right)-I\left(\mathbf{z}_{1}^{k} ;\left(\mathbf{x}(1), \mathbf{w}_{1}^{k}\right) \mid \mathbf{u}_{1}^{k}\right)
$$

It follows from (P2), (P4) and assumption (A7) that:

$$
I\left(\mathbf{u}_{1}^{k} ;\left(\mathbf{x}(1), \mathbf{w}_{1}^{k}\right) \mid \mathbf{z}_{1}^{k}\right)=I\left(\mathbf{u}_{\alpha+1}^{k} ;\left(\mathbf{x}(1), \mathbf{w}_{1}^{k}\right) \mid \mathbf{z}_{1}^{k}, \mathbf{u}_{1}^{\alpha}\right)+I\left(\mathbf{u}_{1}^{\alpha} ;\left(\mathbf{x}(1), \mathbf{w}_{1}^{k}\right) \mid \mathbf{z}_{1}^{k}\right)=I\left(\mathbf{u}_{1}^{\alpha} ;\left(\mathbf{x}(1), \mathbf{w}_{1}^{k}\right) \mid \mathbf{z}_{1}^{k}\right)
$$

Substitution of (83) in (82), together with property (P1), leads to:

$$
I\left(\mathbf{u}_{1}^{k} ;\left(\mathbf{x}(1), \mathbf{w}_{1}^{k}\right)\right) \leq I\left(\mathbf{z}_{1}^{k} ;\left(\mathbf{x}(1), \mathbf{w}_{1}^{k}\right)\right)+I\left(\mathbf{u}_{1}^{\alpha} ;\left(\mathbf{x}(1), \mathbf{w}_{1}^{k}\right) \mid \mathbf{z}_{1}^{k}\right)
$$

Accordingly, (84) and the assumption (A8), which requires $\lim \sup _{k \rightarrow \infty} \frac{1}{k} I\left(\mathbf{u}_{1}^{\alpha} ;\left(\mathbf{x}(1), \mathbf{w}_{1}^{k}\right) \mid \mathbf{z}_{1}^{k}\right)=$ 0 , imply that:

$$
\limsup _{k \rightarrow \infty} \frac{1}{k} I\left(\mathbf{u}_{1}^{k} ;\left(\mathbf{x}(1), \mathbf{w}_{1}^{k}\right)\right) \leq \limsup _{k \rightarrow \infty} \frac{1}{k} I\left(\mathbf{z}_{1}^{k} ;\left(\mathbf{x}(1), \mathbf{w}_{1}^{k}\right)\right)
$$

which, together with (81), concludes the proof.

The following is the statement of the main theorem of Chapter 5 of [10], repeated here for convenience:

Theorem 2.2: (Reproduced from [10], pp.64-65) Let $\hat{F}_{e}(\omega)$ be a real-valued function of the class $\mathcal{L}_{1}\left(\left|\hat{F}_{e}(\omega)\right|\right.$ is integrable in the sense of Lebesgue). We denote by $m$ and $M$ the essential lower bound and upper bound of $\hat{F}_{e}(\omega)$, respectively, and assume that $\mathrm{m}$ and $\mathrm{M}$ are finite. If $G(\lambda)$ is any continuous function defined in the finite interval $m \leq \lambda \leq M$, we have:

$$
\lim _{k \rightarrow \infty} \frac{\sum_{i=1}^{n} G\left(\lambda_{i}\left(\Sigma\left(\mathbf{e}_{1}^{k}\right)\right)\right)}{k+1}=\frac{1}{2 \pi} \int_{-\pi}^{\pi} G\left(\hat{F}_{e}(\omega)\right) d \omega
$$




\section{APPENDIX III}

\section{MeAsure Theoretic Aspects of Differential Entropy}

Proposition 3.1: If a is a random variable with a probability density $p_{a} \in \overline{\mathbb{L}}^{q}$ then $h(a)<\infty$. Proof:(By contradiction) We start by noticing that $p_{a} \in \overline{\mathbb{L}}^{q}$ implies that:

$$
\int_{\mathbb{R}^{q}} p_{a}(\gamma) \gamma^{T} \gamma d \gamma<\infty
$$

If we assume that $h(a)=\infty$ then we should have:

$$
\int_{p_{a}(\gamma) \leq 1}-p_{a}(\gamma) \log p_{a}(\gamma) d \gamma=\infty
$$

Since $p_{a}$ is bounded, (88) also implies that:

$$
\int_{\Xi}-p_{a}(\gamma) \log p_{a}(\gamma) d \gamma=\infty, \text { with } \Xi=\left\{\gamma: p_{a}(\gamma) \leq 1, p_{a}(\gamma) \gamma^{T} \gamma<\left(\gamma^{T} \gamma\right)^{-q / 2},\left(\gamma^{T} \gamma\right)^{1+q / 2}>e\right\}
$$

where we used the fact that $p_{a}$ is bounded and $\mathbb{R}^{q} \backslash \Xi$ has finite volume. On the other hand, $-p \log p$ is an increasing function of $p$ for $p<1 / e$ and $p(\gamma)<\frac{1}{\left(\gamma^{T} \gamma\right)^{1+q / 2}}<e$ for $\gamma \in \Xi$. These facts imply that $-p_{a}(\gamma) \log p_{a}(\gamma) \leq \frac{\log \left(\left(\gamma^{T} \gamma\right)^{1+q / 2}\right)}{\left(\gamma^{T} \gamma\right)^{1+q / 2}}$ for $\gamma \in \Xi$, but $\int_{\Xi} \frac{\log \left(\left(\gamma^{T} \gamma\right)^{1+q / 2}\right)}{\left(\gamma^{T} \gamma\right)^{1+q / 2}} d \gamma<\infty$ holds, thus reaching a contradiction.

Lemma 3.2: (Mutual information expressed by means of differential entropy) Let $\mathbf{a}$ and $\mathbf{b}$ be random variables that admit $p_{a} \in \overline{\mathbb{L}}^{q}, p_{b} \in \overline{\mathbb{L}}^{q^{\prime}}$ and $p_{a, b} \in \overline{\mathbb{L}}^{q+q^{\prime}}$, defined in $\mathcal{A} \times \mathcal{B}=\mathbb{R}^{q} \times \mathbb{R}^{q^{\prime}}$.

The following holds:

$$
I(\mathbf{a} ; \mathbf{b})=h(\mathbf{a})+h(\mathbf{b})-h(\mathbf{a}, \mathbf{b})
$$

\section{Proof:}

Fact1: We start by noticing the fact that if $\mathbf{r}$ is a random variable with $p_{r}$ bounded by $p_{r}^{s u p}$ then the following holds:

$$
\int_{\mathcal{R}}\left[p_{r}\left(\gamma_{r}\right) \log \left(p_{r}\left(\gamma_{r}\right)\right)\right]_{+} d \gamma_{r}<\log \left(p_{r}^{\text {sup }}\right)
$$

which, together with $h(\mathbf{r})<\infty$, implies that $p_{r}\left(\gamma_{r}\right) \log \left(p_{r}\left(\gamma_{r}\right)\right)$ is Lebesgue integrable (see lemma 5 of [1]).

From fact 1, we can use proposition 3.1, of this Appendix, to conclude that $p_{a, b} \log p_{a, b}$, $p_{a} \log p_{a}$ and $p_{b} \log p_{b}$ are Lebesgue integrable. As such, using Theorem 7 of [1], we can split the integral of Theorem 2.1.2. of [17] into a sum of three terms as in (90).

Lemma 3.3: Let the following assumptions hold: 
- $\mathbf{u} \in \mathbb{D}$

- $p_{w_{1}^{k}, \breve{u}_{1}^{k}, x(1)} \in \mathbb{L}^{2 k+1}$

Under the above assumptions, the following holds:

$$
h\left(\mathbf{w}(k) \mid \mathbf{w}_{1}^{k-1}, \mathbf{u}_{1}^{k}, \mathbf{x}(1)\right)=h\left(\mathbf{e}(k) \mid \mathbf{e}_{1}^{k-1}, \mathbf{u}_{1}^{k}, \mathbf{x}(1)\right)
$$

Proof: Since $\mathbf{u} \in \mathbb{D}$, we can use (P3') to write:

$$
\begin{aligned}
h\left(\mathbf{w}(k) \mid \mathbf{w}_{1}^{k-1}, \mathbf{u}_{1}^{k}, \mathbf{x}(1)\right)=h(\mathbf{w}(k))-I\left(\mathbf{w}(k) ;\left(\mathbf{w}_{1}^{k-1}, \mathbf{u}_{1}^{k}, \mathbf{x}(1)\right)\right)= & \\
& h(\mathbf{w}(k))-I\left(\mathbf{w}(k) ;\left(\mathbf{w}_{1}^{k-1}, \breve{\mathbf{u}}_{1}^{k}, \mathbf{x}(1)\right)\right)
\end{aligned}
$$

Since $p_{w_{1}^{k}, \breve{u}_{1}^{k}, x(1)} \in \mathbb{L}^{2 k+1}$, using the change of variables $e(k)=w(k)+u(k)$ and integration, we can show that $p_{w_{1}^{k-1}, \breve{u}_{1}^{k}, x(1)} \in \overline{\mathbb{L}}^{2 k}, p_{e_{1}^{k}, \breve{u}_{1}^{k}, x(1)} \in \overline{\mathbb{L}}^{2 k+1}, p_{e_{1}^{k-1}, \breve{u}_{1}^{k}, x(1)} \in \overline{\mathbb{L}}^{2 k}$ and $p_{e(k)} \in \overline{\mathbb{L}}^{1}$. Accordingly, we can use lemma 3.2, of this appendix, to express (93) as:

$$
h\left(\mathbf{w}(k) \mid \mathbf{w}_{1}^{k-1}, \mathbf{u}_{1}^{k}, \mathbf{x}(1)\right)=h\left(\mathbf{w}_{1}^{k}, \breve{\mathbf{u}}_{1}^{k}, \mathbf{x}(1)\right)-h\left(\mathbf{w}_{1}^{k-1}, \breve{\mathbf{u}}_{1}^{k}, \mathbf{x}(1)\right)
$$

which, by means of the change of variables ${ }^{12} e(k)=w(k)+u(k)$, leads to:

$$
h\left(\mathbf{w}(k) \mid \mathbf{w}_{1}^{k-1}, \mathbf{u}_{1}^{k}, \mathbf{x}(1)\right)=h\left(\mathbf{e}_{1}^{k}, \breve{\mathbf{u}}_{1}^{k}, \mathbf{x}(1)\right)-h\left(\mathbf{e}_{1}^{k-1}, \breve{\mathbf{u}}_{1}^{k}, \mathbf{x}(1)\right)
$$

Similarly, we can use lemma 3.2, of this appendix, to re-express (95) as:

$$
\begin{aligned}
h\left(\mathbf{w}(k) \mid \mathbf{w}_{1}^{k-1}, \mathbf{u}_{1}^{k}, \mathbf{x}(1)\right)=h(\mathbf{e}(k))-I\left(\mathbf{e}(k) ;\left(\mathbf{e}_{1}^{k-1}, \breve{\mathbf{u}}_{1}^{k}, \mathbf{x}(1)\right)\right) \underset{\left(P 3^{\prime}\right)}{=} & \\
& h(\mathbf{e}(k))-I\left(\mathbf{e}(k) ;\left(\mathbf{e}_{1}^{k-1}, \mathbf{u}_{1}^{k}, \mathbf{x}(1)\right)\right)
\end{aligned}
$$

which, from the definition of conditional differential entropy, concludes the proof.

Lemma 3.4: Let $V \in \mathbb{R}^{m \times k-k_{0}+1}$ be a full row-rank matrix. Assume that the following assumptions are satisfied:

- $\mathbf{u} \in \mathbb{D}$

- $p_{w_{1}^{k}, \breve{u}_{1}^{k}, x(1)} \in \mathbb{L}^{2 k+1}$

Under the assumptions above, the following holds ${ }^{13}$ :

$$
h\left(V \mathbf{w}_{k_{0}}^{k} \mid V \mathbf{u}_{k_{0}}^{k}\right)=h\left(V \mathbf{e}_{k_{0}}^{k} \mid V \mathbf{u}_{k_{0}}^{k}\right)
$$

\footnotetext{
${ }^{12}$ Notice that this change of variables is legitimate because we can recover $u(k)$ from $\breve{u}(k)$. Such fact results from the existence of the inverse projection $u(k)=\pi_{\mathcal{U}} \breve{u}(k)$

${ }^{13}$ Here we adopt an abuse of notation by using $V e_{k_{0}}^{k}, V u_{k_{0}}^{k}$ and $V w_{k_{0}}^{k}$ to denote matrix multiplication. For instance, $V e_{k_{0}}^{k}=$ $V\left[\begin{array}{lll}e(k) & \cdots & e\left(k_{0}\right)\end{array}\right]^{T}$.
} 
Proof: The proof of this lemma is concluded by following the same steps of lemma 3.3, mutatismutandis by means of the transformation $V e_{k_{0}}^{k}=V u_{k_{0}}^{k}+V w_{k_{0}}^{k}$.

Lemma 3.5: Let the following assumptions hold:

- $\mathbf{u} \in \mathbb{D}$

- $p_{w_{1}^{k}, \breve{u}_{1}^{k}, x(1)} \in \mathbb{L}^{2 k+1}$

Given a Lipschitz function $f: \mathbb{R}^{k} \rightarrow \mathbb{R}^{\operatorname{dim}\left(x_{u}(1)\right)}$, under the assumptions above, the following holds:

$$
h\left(\mathbf{x}_{u}(1) \mid \mathbf{e}_{1}^{k}\right)=h\left(\mathbf{x}_{u}(1)-f\left(\mathbf{e}_{1}^{k}\right) \mid \mathbf{e}_{1}^{k}\right)
$$

Proof: Since $f$ is Lipschitz, we know that if $\left(\mathbf{w}_{1}^{k}, \mathbf{u}_{1}^{k}, \mathbf{x}(1)\right)$ has a finite covariance matrix then $\left(\mathbf{w}_{1}^{k}, \mathbf{u}_{1}^{k}, \mathbf{x}_{u}(1)-f\left(\mathbf{e}_{1}^{k}\right)\right)$ also has a finite covariance matrix. As such, we can use changes

of variables and integration to show that $p_{w_{1}^{k}, \breve{u}_{1}^{k}, x(1)} \in \mathbb{L}^{2 k+1}$ implies $p_{e_{1}^{k}, x_{u}(1)} \in \overline{\mathbb{L}}^{k+\operatorname{dim}\left(x_{u}(1)\right)}$, $p_{e_{1}^{k}, x_{u}(1)-f\left(\mathbf{e}_{1}^{k}\right)} \in \overline{\mathbb{L}}^{k+\operatorname{dim}\left(x_{u}(1)\right)}, p_{x_{u}(1)} \in \overline{\mathbb{L}}^{k+\operatorname{dim}\left(x_{u}(1)\right)}, p_{e_{1}^{k}} \in \overline{\mathbb{L}}^{k}$ and $p_{x_{u}(1)-f\left(\mathbf{e}_{1}^{k}\right)} \in \overline{\mathbb{L}}^{\operatorname{dim}\left(x_{u}(1)\right)}$. These facts allow us to use lemma 3.2 freely to write:

$$
h\left(\mathbf{x}_{u}(1) \mid \mathbf{e}_{1}^{k}\right)=h\left(\mathbf{x}_{u}(1), \mathbf{e}_{1}^{k}\right)-h\left(\mathbf{e}_{1}^{k}\right)
$$

By applying a change of variables in (99), we get:

$$
h\left(\mathbf{x}_{u}(1) \mid \mathbf{e}_{1}^{k}\right)=h\left(\mathbf{x}_{u}(1)-f\left(\mathbf{e}_{1}^{k}\right), \mathbf{e}_{1}^{k}\right)-h\left(\mathbf{e}_{1}^{k}\right)
$$

We finish by recognizing that (100) is equal to $h\left(\mathbf{x}_{u}(1)-f\left(\mathbf{e}_{1}^{k}\right) \mid \mathbf{e}_{1}^{k}\right)$

\section{ACKNOWLEDGMENT}

The authors would like to thank Prof. Nicola Elia (Iowa State University) for interesting suggestions. The first author is grateful to Prof. John Doyle (Caltech) for his enthusiastic support. We also would like to thank Prof. Sanjoy Mitter for interesting discussions on related problems. This work was sponsored by the University of California - Los Angeles, MURI project title: "Cooperative Control of Distributed Autonomous Vehicles in Adversarial Environments", award: 0205-G-CB222. Nuno C. Martins was partially supported by the Portuguese Foundation for Science and Technology and the European Social Fund, PRAXIS BD19630/99. 


\section{REFERENCES}

[1] Adams, M.; Guillemin, V. Measure Theory and Probability”, Birkhauser, 1996

[2] Billingsley, P. "Probability and Measure”, Wiley Series in Probability and Mathematical Statistics, Third Edition, 1995

[3] Bode, H. W., “Network Analysis and Feedback Amplifi er Design”, D. Van Nostrand, Princeton, 1945

[4] Cover, T.M; Thomas, J. A.; “Elements of Information Theory”, Wiley-Iterscience Publication, 1991

[5] Doyle, J.C.;Francis, B.A.; Tannenbaum, A.R.; “Feedback Control Theory”, Macmillan, New York, 1992

[6] Elia, N., "When Bode meets Shannon: Control-Oriented feedback communication schemes”, IEEE TAC Vol 49, No 9, pp. 1477, September 2004

[7] Fernandez, P. J. “Medida e Integração”, Instituto de Matemática Pura e Aplicada, Projeto Euclides, Segunda Edição, 1996

[8] Freudenberg, J.S.; Looze, D.P. “ Frequency Domain Properties of Scalar and Multivariable Systems”, Springer-Berlin, 1988

[9] Gray, Robert M.; “Probability, Random Processes, and Ergodic Properties”, Springer Verlag, 1988

[10] Grenander, U.; Szego, G.; “Toeplitz Forms and Their Applications”, University of California Press, 1958

[11] Liberzon, D. “ On Stabilization of Non-Linear Systems With Limited Information Feedback”, Proc. of the IEEE Conf. on Dec. and Control , pp. 182-6, 2003

[12] Martins, N. C. “Information Theoretic Aspects of the Control and Mode Estimation of Stochastic Systems”, MIT, Ph.D. Thesis, August 2004

[13] Martins, N.C.; Dahleh, M. A. and Elia, N.; "Feedback Stabilization of Uncertain Systems Using a Stochastic Digital Link”, to appear in the IEEE Conf.on Decision and Control, 2004

[14] Massey, J. “Causality, Feedback and Directed Information”, Proc. of the 1990 Int. Symp. on Information Theory and its Applications (ISITA-90), pp. 303-305

[15] Nair, G. N. and Evans, R. J., "Stabilizability of Stochastic Linear Systems with Finite Feedback Data Rates” SIAM J. Control Optim. Vol 43, No 2, pp. 413-436, 2004

[16] Nair, G. N. and Evans, R. J., Mareels, I. M. Y. and Moran W. “ Topological Entropy and Nonlinear Stabilization”, IEEE TAC Vol 49, September 2004

[17] Pinsker, M. S.; “Information and Information Stability of Random Variables and Processes”, Holden Day, 1964

[18] Sahai, A.; “Evaluating Channels for Control: Capacity Reconsidered”, Proc. ACC., pp. 2358 - 2362, 2000

[19] Tatikonda, S.; "Control under Communication Constraints: Part I and II", submitted to the IEEE Transactions on Automatic Control

[20] Tatikonda, S.; “Control under Communication Constraints”, Ph.D. Thesis, M.I.T. 2000

[21] Witsenhausen, H., "Separation of Estimation and Control for Discrete-Time Systems", Proceeding of the IEEE, Volume 59, No 11, November 1971

[22] Wong, W.S.; Brockett, R.W.; "Systems with fi nite communication bandwidth constraints -I: State estimation problems" IEEE Trans. Automat. Control, Vol 42, pp. 1294-1298, Sept 1997

[23] Wong, W.S.; Brockett, R.W.; “Systems with fi nite communication bandwidth constraints -II: Stabilization with Limited Information Feedback” IEEE Trans. Automat. Control, Vol 44, No. 5 pp. 1049-1053, 1999

[24] Yuksel, S.; Basar, T. “Quantization and Coding for Decentralized LTI Systems”, Proc. IEEE CDC, Hawai, December 2003

[25] Zang, G.;Iglesias, P. A., “Nonlinear extension of Bode's integral based on an information theoretic interpretation”, Systems and Control Letters, 50 (2003) pp. 11-19 